\title{
Theoretical Implications of Articulatory Duration, Phonological Similarity, and Phonological Complexity in Verbal Working Memory
}

\author{
Shane T. Mueller, Travis L. Seymour, David E. Kieras, and David E. Meyer \\ University of Michigan
}

\begin{abstract}
The phonological-loop model provides a prominent theoretical description of verbal working memory. According to it, serial recall accuracy should be inversely related to the articulatory duration and phonological similarity of verbal items in memorized sequences. Initial tests of these predictions by A. D. Baddeley and colleagues (e.g., A. D. Baddeley, N. Thomson, \& M. Buchanan, 1975) appeared to support the phonological-loop model, but subsequent researchers have obtained conflicting data that putatively disconfirm its assumptions. Such conflicts may have stemmed from less than ideal measurements of articulatory duration and phonological similarity. This article discusses these concerns and proposes new theoretically principled methods for measuring articulatory duration and phonological similarity. Two experiments that used these methods in the context of a verbal serial recall task are reported. The results of these experiments confirm and extend the predictions of the phonological-loop model while disarming previous criticisms of it.
\end{abstract}

Since Miller's (1956) classic article on "the magical number seven, plus or minus two," short-term verbal working memory (VWM) has become an increasingly important topic of investigation in experimental psychology and cognitive science. VWM plays a major role during performance of many basic mental tasks, ranging from serial recall to sentence comprehension, syllogistic reasoning, and arithmetic problem solving (Baddeley, 1986; Baddeley, 1992; Baddeley \& Logie, 1999). Consequently, to account for such performance, investigators have proposed numerous hypotheses about the mechanisms that mediate VWM (e.g., Anderson \& Matessa, 1997; Baddeley \& Hitch, 1974; Brown \& Hulme, 1995; Cantor \& Engle, 1993; Cowan, 1999; Dosher \& Ma, 1998; Drewnowski, 1980; Estes, 1972; Kieras, Meyer, Mueller, \& Seymour, 1999; Laughery \& Pinkus, 1970; Lewandowsky \& Murdock, 1989; Murdock, 1993; Nairne, 1990; Page \& Norris, 1998; Sperling, 1967; Sternberg, Monsell, Knoll, \& Wright, 1978; Waugh \& Norman, 1965).

Shane T. Mueller, Travis L. Seymour, and David E. Meyer, Cognition and Perception Program, Department of Psychology, University of Michigan; David E. Kieras, Department of Electrical Engineering and Computer Science, University of Michigan.

Travis L. Seymour is now at the Department of Psychology, University of California, Santa Cruz.

Funding for this research was provided by United States Office of Naval Research Grant N00014-92-J-1173 to the University of Michigan.

We thank members of the Brain, Cognition and Action Laboratory at the University of Michigan (David Fencsik, Darren Gergle, Jennifer Glass, Leon Gmeindl, Cerita Jones, Eric Schumacher, Adam Krawitz, and Mollie Schweppe) for helpful comments, and Beth Emmons, Sean Ferguson, Michelle Gayewski, Ryan Kettler, Mollie Schweppe, and Michaela Ferguson for assistance in conducting the experiments. Reviewer suggestions by Nelson Cowan and Gary Dell are gratefully acknowledged.

Correspondence concerning this article should be addressed to Shane T. Mueller or David E. Meyer, Cognition and Perception Program, Department of Psychology, University of Michigan, 525 East University, Ann Arbor, Michigan 48109-1109. E-mail: smueller@umich.edu or demeyer@ umich.edu
Following these proposals, significant controversies have arisen about which hypotheses best explain and predict various empirical phenomena related to VWM (Miyake \& Shah, 1999). A major focus of controversy has been the influential phonological-loop model of VWM proposed by Baddeley (1986, 1992) and his colleagues (Baddeley \& Hitch, 1974; Baddeley, Lewis, \& Vallar, 1984; Baddeley, Thomson, \& Buchanan, 1975). The basis for this controversy concerns the putative effects of phonological complexity (i.e., the numbers of phonemes and syllables in a word) ${ }^{1}$ and articulatory duration (i.e., the time taken to pronounce a word) on performance of the verbal serial recall task. Critics of the phonological-loop model have argued that its accounts of these effects are either incomplete or incorrect (e.g., Caplan, Rochon, \& Waters, 1992; Caplan \& Waters, 1994; Cowan, Nugent, Elliot, \& Geer, 2000; Cowan, Wood, Nugent, \& Treisman, 1997; Cowan et al., 1998; Lovatt, Avons, \& Masterson, 2000; Service, 2000). In reply, proponents of the phonological-loop model have claimed that such arguments are dubious and do not require its assumptions to be substantially modified or discarded (e.g., Baddeley \& Andrade, 1994; Baddeley \& Logie, 1999).

We believe that this controversy has stemmed at least partly from less than ideal measurement and control of factors such as articulatory duration, phonological similarity, and phonological complexity. Thus, the purpose of this article is to consider how these factors have been measured in the past and to propose new methodological techniques for measuring them that may help resolve some of the persistent controversies about their roles in VWM tasks. Our approach here includes the following steps: First, we describe the phonological-loop model of VWM. Second, we discuss several recent empirical studies that have attempted to

\footnotetext{
${ }^{1}$ The term phonological complexity (as used by Service, 1998), which we have adopted here, should not be confused with the term articulatory complexity (as used by Caplan et al., 1992). Articulatory complexity can be thought of as indexing ease of articulation and is therefore more closely related to articulatory duration than is phonological complexity, which indexes the size of the sublexical phonological representation for a word.
} 
measure the effects of articulatory duration, phonological similarity, and phonological complexity on VWM. Third, we introduce more appropriate theoretically principled methods that can be used for measuring phonological similarity and articulatory duration. Fourth, we present results of new experiments that confirm the utility and informativeness of our methods. Fifth, we evaluate the status of the phonological-loop model on the basis of our findings.

\section{The Phonological-Loop Model}

Several related versions of the phonological-loop model have been proposed (e.g., Baddeley, 1986, 1992; Baddeley \& Hitch, 1974; Baddeley et al., 1984; Sperling, 1967). The one that we consider here comes mainly from Baddeley (1986). This model has three interconnected components: the phonological short-term store, the articulatory motor-program processor, and the central executive. The phonological short-term store is a repository of temporary phonological codes for verbal items. The articulatory motor-program processor enables the phonological codes to be refreshed through a cyclic rehearsal process. During its operations, codes in the phonological short-term store are converted sequentially to articulatory motor programs and vocalized overtly or covertly one after another. Finally, the central executive supervises interactions between the articulatory motor-program processor and phonological short-term store as well as other working-memory components.

\section{Empirical Support}

Support for the phonological-loop model has come from experiments with a wide range of tasks that stress VWM. Although many variables are known to affect VWM, we focus primarily on the effects of two factors: word length and phonological similarity.

The word-length effect. ${ }^{2}$ Some researchers have found that serial recall accuracy is better for sequences of words whose articulatory durations are shorter (e.g., Baddeley et al., 1975; Schweickert \& Boruff, 1986; Longoni, Richardson, \& Aiello, 1993). Baddeley et al. (1975, Experiment IV) investigated this effect by comparing serial recall accuracy for two sets of words that had different mean articulatory durations but equal phonological complexity (i.e., numbers of phonemes and syllables). They found that sequences with shorter durations were remembered better than sequences with longer durations.

According to the phonological-loop model, articulatoryduration effects occur because words that are spoken more rapidly can be rehearsed more frequently. Supposedly, words that are rehearsed more frequently are less likely to decay before an entire sequence of them can be recalled. Thus, serial recall accuracy for sequences of shorter words should tend to be higher than that for sequences of longer words.

Phonological-similarity effect. Another important factor that influences serial recall accuracy is phonological similarity. Typically, sequences of phonologically similar words are remembered less well than sequences of dissimilar words (e.g., Baddeley, 1966, 1968; Conrad \& Hull, 1964; Schweickert, Guentert, \& Hersberger, 1990). The phonological-similarity effect supports the phonological-loop model's assumption that verbal information is represented in a modality-specific phonological store rather than in another type of storage system such as a visual or semantic buffer.
Under the phonological-loop model, there are at least two explanations of the phonological-similarity effect. One possibility is that during rehearsal and recall, phonological codes decay more quickly when they are similar to each other (Posner \& Konick, 1966). Another possibility is that the codes decay at the same rate regardless of their similarity, but at the time of recall, the partially degraded codes of similar items are more difficult to reconstruct or redintegrate (Hulme et al., 1997; Nairne, 1990). Either of these possibilities could yield an inverse relationship between recall accuracy and phonological similarity in the serial recall task.

Other factor effects. Many other factors may also affect performance in VWM tasks. These factors include unattended irrelevant speech (e.g., Colle \& Welsh, 1976; Salamé \& Baddeley, 1982), rehearsal strategy (e.g., Cowan et al., 1997; Logie, Della Sala, Laiacona, \& Chalmers, 1996; Standing, Bond, Smith, \& Isely, 1980; Standing \& Curtis, 1989), articulatory suppression (e.g., Baddeley et al., 1975; Levy, 1971; Murray, 1967, 1968), and focal brain damage (e.g., Baddeley \& Wilson, 1985; Basso, Spinnler, Vallar, \& Zanobio, 1982; D’Esposito \& Postle, 2000; Della Sala, Logie, Marchetti, \& Wynn, 1991; Shallice \& Warrington, 1970). Research on the effects of these factors has provided considerable evidence concerning the structure of VWM. This evidence has, for the most part, supported the assumptions of the phonological-loop model. However, for present purposes, the effects of word length and phonological similarity are most directly relevant to the prevailing criticisms of the phonological-loop model

\section{Criticisms of the Phonological-Loop Model}

Despite the success of the phonological-loop model, it has become the target of many critiques by researchers who claim that its assumptions are incorrect. Consequently, there are now probably more experiments whose results appear to contradict Baddeley's (1986, 1992) conclusions about the word-length effect than there are experiments that support them. Some of the first such critical experiments were reported by Caplan, Rochon, and Waters (1992).

Caplan et al. (1992). On the basis of studies of neuropsychological patients, Caplan et al. hypothesized that the word-length effect stems from speech planning rather than overt or covert articulation. Their hypothesis assumed that speech-planning times are influenced by the phonological complexity of words, as indexed by numbers of phonemes and syllables. ${ }^{3}$ Caplan et al. noted that most of the evidence for the word-length effect has come from

\footnotetext{
${ }^{2}$ Both articulatory duration and phonological complexity can be used to specify a word's length, making the term word-length effect ambiguous. To avoid this ambiguity, we use either articulatory duration or phonological complexity to specify the particular aspect of word length being considered at the moment. However, when the source of the effect is unknown or controversial, we use the more general term word length.

${ }^{3}$ Although the speech-planning hypothesis may seem plausible, it should be taken with some reservations. Not all empirical evidence is consistent with it. For example, Sternberg et al. (1978) had participants utter memorized sequences of either one-syllable or two-syllable words on command. For sequences that contained one to five words each, the latencies of these utterances in response to a go signal were not affected reliably by the phonological complexity (i.e., syllable numerosity) of the individual words. Given that the onset latency of an utterance may manifest speech-planning times (Rosenbaum, Gordon, Stillings, \& Feinstein, 1987; cf. Sternberg et al., 1978), these results are inconsistent with Caplan et al.'s (1992) hypothesis and assumptions.
} 
Table 1

Articulatory Durations of Short and Long Words Measured in Previous Studies of VWM

\begin{tabular}{llll}
\hline & & \multicolumn{2}{c}{$\begin{array}{c}\text { Mean articulatory duration per word } \\
\text { S(ms) }\end{array}$} \\
\cline { 3 - 4 } Source & Articulated items & Long words & Short words \\
\hline Caplan et al. (1992) & & & \\
Exp. 2 & Individual words* & 720 & 546 \\
Baddeley \& Andrade (1994) & & 360 & 353 \\
Exp. 1 & Overt word pairs & 364 & 353 \\
Exp. 2 & Overt word pairs* & 331 & 320 \\
Exp. 2 & Covert word pairs & 525 & 473 \\
Caplan \& Waters (1994) & Overt word lists* & 571 & 444 \\
Lovatt et al. (2002) & Individual words* & 433 & 343 \\
$\quad$ Exp. 4 & Recall durations* & & \\
Exp. 4 & & & \\
\hline
\end{tabular}

Note. $\quad \mathrm{VWM}=$ verbal working memory; Exp. = experiment.

${ }^{a}$ Values were obtained by dividing reported mean total articulation times by 20 (the number of words in 10 repetitions of word pairs). ${ }^{\mathrm{b}}$ Values were obtained by dividing mean total articulation times by 5.05 , the average list length.

$* p<.05$

cases in which articulatory duration and phonological complexity were confounded, and they dismissed the few cases in which these confounds did not occur (e.g., Experiment IV of Baddeley et al., 1975).

To support their hypothesis and assumptions, Caplan et al. (1992) performed three experiments with various sets of words. Each experiment involved versions of the immediate serial recall task in which stimuli were presented either auditorily or visually, and responses were made with a "picture-pointing" procedure. They measured articulatory durations for each word set by recording words spoken in isolation by a confederate speaker. Computer software was used to determine the acoustic onset and offset of each word.

Experiment 1 of Caplan et al. (1992) yielded typical effects of both articulatory duration and phonological similarity: Sequences of one-syllable words were recalled more accurately than sequences of three-syllable words, and sequences of dissimilar words were recalled more accurately than sequences of similar words. However, this pattern failed to occur in their Experiments 2 and 3. During Caplan et al.'s Experiment 2, to-be-recalled sequences were constructed from two new word sets that had either long or short articulatory durations but approximately equal phonological complexity. Contrary to Experiment 1, results of Experiment 2 revealed that sequences from the long set were remembered better than sequences from the short set. Furthermore, during Caplan et al.'s Experiment 3, sequences were constructed from two new word sets that were either difficult or easy (with long and short articulatory durations, respectively) but with approximately equal phonological complexity. Results of Experiment 3 revealed that sequences from the difficult word set were remembered as well as sequences from the easy word set, contrary to both Experiments 1 and 2 .

As a result, over these three experiments, the data appeared to seriously challenge the phonological-loop model. In them, a wordlength effect consistent with this model occurred only in Experiment 1 , in which articulatory duration was confounded with phonological complexity. In Experiments 2 and 3, phonological complexity was equated across word sets, but the model's predictions were not substantiated. Consequently, Caplan et al. (1992) proposed that the word-length effect does not stem from articulatory rehearsal but from rehearsal through speech planning based on sublexical phonemic representations whose duration varies with the numbers of phonemes and syllables per word. Yet they did not explain why the results from their Experiments 2 and 3 differed.

Baddeley and Andrade (1994). In reply, Baddeley and Andrade (1994) criticized these experiments by claiming there were two problems with Caplan et al.'s (1992) word sets: Their articulatory durations had not been measured properly, and they were not equal in phonological similarity.

To support their claims, Baddeley and Andrade (1994) measured the articulatory durations for the words of the two sets in Caplan et al.'s (1992) Experiment 2. ${ }^{4}$ These additional measurements were obtained by having participants rapidly repeat pairs of words 10 times, either overtly or covertly. This procedure was similar to one used by Baddeley et al. (1975) but differed from the isolated word measurement procedure used by Caplan et al. As a result, there were only slight differences between the articulatory durations for the two sets of words (see Table 1). This led Baddeley and Andrade to argue that the obtained duration differences were too small (i.e., less than $15 \mathrm{~ms}$ per word) to justify rejecting the phonological-loop model.

Baddeley and Andrade (1994) also obtained participants' judgments about the phonological similarity for the word sets used by Caplan et al. (1992, Experiment 2). Here the short words were rated as more phonologically similar than the long words. This suggests that Caplan et al.'s results stemmed, at least in part, from systematic confounded differences between phonological similarity and articulatory duration.

Caplan and Waters (1994). Caplan and Waters (1994) responded to Baddeley and Andrade's (1994) criticisms by repeating Experiments 2 and 3 from Caplan et al. (1992). In this replication,

\footnotetext{
${ }^{4}$ Only Caplan et al.'s (1992) Experiment 2 was addressed because the results of their Experiment 1 were not controversial, and the results of their Experiment 3 were not replicated subsequently by Caplan and Waters (1994).
} 
articulatory durations were measured by recording participants' speech while they read the actual word lists used in the serial recall task. Results like those of Caplan et al.'s original Experiment 3 were not obtained here: Sequences of longer words from this experiment were remembered less well than sequences of shorter words, contrary to their speech-planning hypothesis. However, results like those of Caplan et al.'s original Experiment 2 were obtained, contradicting the results of Baddeley and Andrade. In Caplan and Waters, long words from Experiment 2 had longer durations than short words (see Table 1), and long words were also recalled better. Caplan and Waters also had participants rate the phonological similarity of their words. They found no reliable difference between the rated similarity for the word sets from Caplan et al.'s Experiment 2, which contradicts Baddeley and Andrade's results.

Consequently, the evidence remains rather equivocal about whether rehearsal in VWM is based on speech planning or on actual articulatory execution. ${ }^{5}$ Caplan and colleagues (Caplan et al., 1992; Caplan \& Waters, 1994) have shown twice that the words in a long set had longer articulatory durations than the words in a short set but that serial recall accuracy was better for sequences of the long words. They also have shown that words in these two short and long sets were about equal in rated phonological similarity. In contrast, Baddeley and Andrade (1994) found that words in these sets did not differ significantly with respect to articulatory duration but did differ with respect to rated phonological similarity. However, their data were produced by a different group of participants who spoke British English, which limits their data's relevance to the results produced by North American participants in the studies of Caplan and colleagues.

Cowan et al. (1997). Other objections have also been raised about the phonological-loop model's account of the word-length effect. In one case, Cowan et al. (1997) explored the extent to which articulatory duration and phonological complexity have separable effects on verbal serial recall accuracy. They conducted an experiment in which participants performed backward recall for sequences of simple one-syllable words or complex two-syllable words. Participants were instructed to recall the words at either a rapid or slow pace, so that the articulatory durations for the simple and complex words would be approximately equated during the recall phase.

As a result of these manipulations, two separate effects emerged. Overall, recall accuracy decreased as articulatory duration during recall increased, consistent with the phonological-loop model. However, when the durations of the simple and complex words were approximately equated during recall, participants recalled the complex words more accurately than the simple words. This latter result cannot be easily explained by either the phonological-loop model or the speech-planning hypothesis of Caplan et al. (1992). Nevertheless, Cowan et al. (1997) concluded that the phonological-loop model was essentially correct but should be supplemented with an assumption that phonological complexity enhances recall accuracy.

Service (1998). A study by Service (1998) also addressed issues regarding serial recall accuracy, articulatory duration, and phonological complexity. She exploited the fact that in Finnish, some words contain double vowels that are identical to single vowels except for their articulatory durations. This enabled her to construct two sets of simple pseudowords that were identical in terms of their phonological complexity and phonological similarity yet differed in their articulatory durations. She also constructed a third set of complex pseudowords that had longer articulatory durations and more phonemes per pseudoword.

Service (1998) found that sequences of simple double-vowel pseudowords were recalled as well as sequences of simple singlevowel pseudowords. Both of these types of sequences were recalled better than sequences of longer, complex pseudowords. To check whether her simple single-vowel and double-vowel pseudowords actually had different articulatory durations, she measured the times taken by participants to read lists of them. Double-vowel pseudowords took $31 \%$ longer to read than lists of single-vowel pseudowords.

These results, which reveal a dissociation between recall accuracy and articulatory duration, raise further doubts about the phonological-loop model. Consequently, Service (1998) argued that phonological complexity influences serial recall accuracy but that articulatory duration and time-based decay do not. She suggested that Cowan et al.'s (1997) findings (i.e., that participants recalled longer words less well than shorter words) were artifactual and that they probably occurred because participants had to perform backward recall at a fixed, unnatural rate.

Cowan, Nugent, Elliot, and Geer (2000). In response to Service (1998), Cowan, Nugent, Elliot, and Geer (2000) conducted a new study that replicated several conditions of the experiments by Cowan et al. (1997). In this replication, participants were trained to recall sequences of simple one-syllable words and sequences of complex two-syllable words at both rapid and slow paces. Unlike in Cowan et al. (1997), forward recall was required here. As a result, recall was more accurate under the rapid-recall condition, but when recall rate was approximately equal, recall was more accurate for the simple words than for the complex words. These results again suggest that articulatory duration does affect serial recall accuracy. However, Cowan et al.'s (1997) earlier conclusion about phonological complexity is called into question, because the complexity effect in the latter experiment was opposite to what had occurred previously.

Service (2000). In reply to Cowan, Nugent, Elliot, and Geer (2000), Service (2000) suggested that their study still had methodological problems. For example, its artificial manipulation of recall rate may have induced atypical effects because participants divided their attention between recalling words and controlling their recall rate, which constituted an unusual dual-task situation. Consequently, Service argued that her original procedure and

\footnotetext{
5 The distinction between speech planning and articulatory execution is important, especially with respect to understanding clinical disorders (e.g., dysarthria) that putatively affect vocal articulation. A primary motivation for Caplan et al.'s (1992) speech-planning hypothesis was to explain certain aspects of serial recall from VWM in patients with dysarthria of speech, who show normal word-length effects even though they have deficient overt articulation. Veridical theories of speech-based rehearsal should account for such effects. Consequently, we define articulatory execution to mean the operation of the articulatory motor-program processor not at a peripheral effector level but rather at a central level, where it produces phonological codes that maintain and update the contents of the phonological buffer (cf. Kieras et al., 1999). This type of covert operation does not require physical movement of the speech articulators, so it may remain intact in patients who have dysarthria of speech. Furthermore, it can account for why these patients show a word-length effect, because implicit (covert) speech occurs at the same rate as overt speech (Landauer, 1962).
} 
theoretical inferences (Service, 1998) remain on solid ground. Subsequently, however, Cowan, Nugent, and Elliot (2000) have disagreed with her argument and continued to advocate their position over Service's (1998, 2000). Insofar as we can tell, this debate has not been satisfactorily resolved to date.

Lovatt et al. (2000). Meanwhile, research by Lovatt et al. (2000) has created yet more uncertainty about the status of the phonological-loop model. They conducted three experiments with two sets of two-syllable words per experiment (including a total of six different word sets). The mean articulatory durations of the words in each set were measured, and subjective ratings of the words' phonological similarity were also obtained. These measurements showed that for each experiment, the articulatory durations of the two word sets differed reliably, and the phonological similarity of the words in the sets was approximately equal. However, Lovatt et al.'s three experiments yielded an inconsistent pattern of results, contrary to the phonological-loop model.

Specifically, in their Experiment 1, Lovatt et al. (2000) measured articulatory duration with a list-reading technique and found that sequences with longer durations were recalled better than sequences with shorter durations. In Experiment 2, using two more word sets, Lovatt et al. obtained three measurements of word length (i.e., isolated word durations, list-reading times, and articulation times for rapidly repeated word pairs). They found that although articulatory durations of the words from the one set were consistently shorter than those from the other set, there was almost no difference in recall accuracy for the two sets. Finally, in Experiment 3, using a third pair of word sets, Lovatt et al. measured articulatory durations with the same three procedures as those of Experiment 2. They found that sequences of shorter duration words were recalled more accurately than sequences of longer duration words. Thus, on balance, there was no consistent relationship between articulatory duration and serial recall accuracy across the word sets of these three experiments. Although Lovatt et al. did not provide any principled theory to explain their results, they imply that the phonological-loop model is probably incorrect.

Lovatt, Avons, \& Masterson (2002). Extending their prior research, Lovatt et al. (2002) conducted four more experiments that compared immediate serial recall for sequences of short and long words. They confirmed that some previous observations of articulatory durations and serial recall accuracy were reliable.

Experiments 1 and 3 by Lovatt et al. (2002) used subsets of the words from Experiment 2 by Lovatt et al. (2000). In the latter experiments, articulatory durations were measured in three ways, which included recording the durations of (a) confederates who read isolated words, (b) experimental participants who read lists of printed words, and (c) participants who spoke repetitive triplets of words. In each of these cases, nominally short words had shorter durations than did long words, as Lovatt et al. (2000) had found before. Also, serial recall accuracy was similar to the previous findings by Lovatt et al. (2000): No reliable differences occurred between recall accuracy for pure sequences of short words and pure sequences of long words. Once again, given that the phonological-loop model predicts recall accuracy should be lower for sequences of long words, these results seem to disconfirm this model.

Experiment 4 by Lovatt et al. (2002) used the short and long words from Caplan et al. (1992, Experiment 2). In this experiment, articulatory durations were measured in two ways, which included speech recordings of (a) confederates reading the words at a normal rate and (b) participants recalling word sequences from memory at the end of experimental recall trials. The mean durations per word on the basis of these measurements appear in Table 1. They show that the nominally short words again had shorter durations than the nominally long words, as in Caplan et al. Also consistent with Caplan et al., Lovatt et al. found that participants recalled sequences of these long words more accurately than sequences of these short words, further contradicting the phonological-loop model's predictions.

In addition, Experiment 2 by Lovatt et al. (2002) included mixed-length word sequences; for them, the first half of each sequence contained short words and the second half contained long words, or vice versa. Performance with such sequences may be helpful for testing hypotheses about the respective roles of decay and output interference in forgetting from VWM. However, in our opinion, this is not directly relevant to resolving the present debate about the ultimate veracity of the phonological-loop model, so for now, we do not further discuss the results from mixed-length sequences.

\section{Interim Assessment}

Although some data from the studies reviewed here appear to offer evidence against the phonological-loop model, together they do not suggest a clear alternative theory because of inconsistencies and contradictions among them. Perhaps these inconsistencies indicate that the phonological-loop model is difficult to test and needs to be specified more precisely. This difficulty is highlighted by the fact that across past tests of the model, experimenters have measured articulatory duration with five distinct methods whose rationale remains unclear. None of these measurements may be adequate for testing the model's predictions about serial recall accuracy. Likewise, in these same experiments, phonological similarity was either disregarded or measured only through informal subjective ratings. Thus, the true effects of this factor may have been frequently confounded with those of articulatory duration and phonological complexity.

In light of these considerations, previous results that appear to contradict the phonological-loop model may reflect just the less than ideal measurement of articulatory duration or phonological similarity. Consequently, to pursue these matters further, we have developed more theoretically principled methods for measuring phonological similarity and articulatory duration, by which predictions of the phonological-loop model and other alternative hypotheses can be precisely tested. In the following sections, we describe our new methods for measuring phonological similarity and articulatory duration. Then we report two experiments with these methods that evaluate the predictions of the phonologicalloop model and the criticisms of its opponents.

\section{Phonological Similarity Metric Analysis (PSIMETRICA): A Formal Method for Measuring Phonological Dissimilarity}

The effect of phonological similarity on serial recall implies that coded information in VWM has a phonological representation (e.g., Baddeley, 1966, 1968). Yet the phonological-loop model makes no explicit claims about the details of this representation. To test this and other models of VWM and serial recall, we have 
developed PSIMETRICA, a formal method for quantifying the phonological dissimilarity of paired words.

PSIMETRICA assumes that the phonological dissimilarity between words is a multidimensional vector of psychologically relevant aspects of dissimilarity, which we call a dissimilarity profile. The individual dimensions of this profile may include a variety of quantities, such as an index of the extent to which two words rhyme, the similarity of their stress patterns, and the degree to which the words' syllable onsets match or mismatch. The use of a dissimilarity profile enables us to quantify various distinct dimensions of dissimilarity and to determine which ones matter more or less in any given situation. In this article, we focus on three such dimensions, each related to syllable onsets, nuclei, and codas (see below). ${ }^{6}$

Application of PSIMETRICA involves four main steps. First, given a set of words, we specify the contents of the phonological representation for each individual word. Second, for each dimension of the dissimilarity profile, we identify and align the phonemes in a pair of words, so that they may be compared to assess how much they match or mismatch. Third, we quantify the degrees of match and mismatch for each dimension of the dissimilarity profile. Fourth, we average the results of this quantification over all of the possible word pairs of the word set. This yields the mean phonological dissimilarity profile for the word set. In the following subsections, these steps are described more fully.

\section{Phonological Representation of a Word}

Figure 1 shows the schematic hierarchical structure that we use for representing English words in PSIMETRICA. Here, a word is assumed to be composed of syllables. Each syllable has several properties (e.g., stress and intonation) and contains three phoneme clusters: the onset (initial consonants), the nucleus (vowels), and the coda (final consonants). The nucleus and coda together are called the rhyme. The onset contains between zero and three consonant phonemes, the nucleus contains either one or two vowel

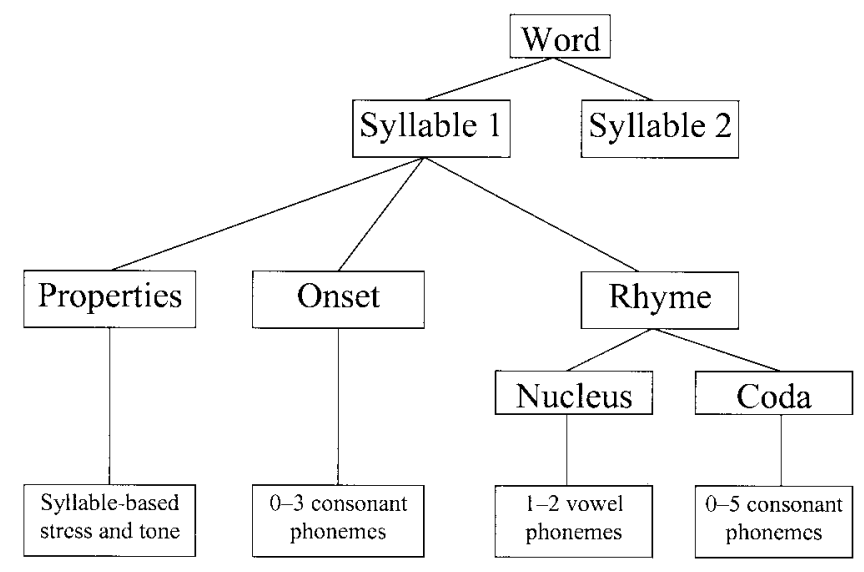

Figure 1. The hierarchical word structure used in Phonological Similarity Metric Analysis for phonological dissimilarity measurement. Here, a word is assumed to be composed of one or more syllables which consist of properties and clusters of phonemes. Phonemes are associated with 13 binary features that describe the articulatory states necessary to produce appropriate speech sounds. phonemes, and the coda contains between zero and five consonant phonemes.

Decomposition of words into syllables and phoneme clusters. To decompose words into their constituent syllables and phoneme clusters, we use a standard linguistic procedure (O'Grady \& Dobrovrolsky, 1992, pp. 76-82). In this procedure, the vowel nucleus of each syllable is identified first. Next, the longest string of consonants to the left of each syllable's nucleus that conforms to phonotactic and linguistic constraints is placed in the syllable's onset. Finally, all remaining consonants to the right of the syllable's nucleus form the coda. This decomposition is made for each syllable of a word, starting from the final syllable and working toward the first syllable. ${ }^{7}$

Decomposition of phonemes into phonological features. Next, for each phoneme in a word's representation, we identify its phonological features, using the system of Chomsky and Halle (1968). Under this system, each phoneme has 13 or fewer binary features that describe the states of certain vocal articulators when they produce the speech sound associated with a phoneme. A list of some common English phonemes and their constituent phonological features appears in Appendix A. On this basis, the phonological representations for any word may be generated.

Illustrative examples. The four steps that would be followed to obtain the phonological representations of the words amount and placemats appear in Table 2. For the word placemats, the syllable boundary is placed between the two constituent words, place and mats, even though the phoneme cluster $/ \mathrm{sm} /$ is a legal onset (see Footnote 7). For amount, the syllable boundary is placed between the phonemes $/ \partial /$ and $/ \mathrm{m} /$. When phonemes are assigned to their appropriate clusters, a null phoneme $/ \varnothing /$ is used as a placeholder for clusters that contain no phonemes (such as the onset of the first syllable in amount).

\section{Alignment Procedures for Individual Dissimilarity Dimensions}

After the phonological representation of each word has been generated, PSIMETRICA's next step aligns these representations according to the constraints associated with each dimension of the dissimilarity profile. These constraints ensure that corresponding phonemes of the paired words are properly compared with each other in terms of whatever psychological hypotheses are embodied

\footnotetext{
${ }^{6}$ Other dimensions of dissimilarity might also be relevant, depending on the task that participants have to perform for the words to which PSIMETRICA is applied. For example, some of these other dimensions might include initial phoneme dissimilarity, stress dissimilarity, intersyllable or intercluster dissimilarity, syllable or phoneme numerosity dissimilarity, phoneme distribution dissimilarity, and so forth. We have not yet exhaustively investigated the effects of all these dimensions in serial recall and other tasks.

${ }^{7}$ Multisyllabic words are frequently composites of smaller words or morphemes (phoneme sequences that carry meaning), and the syllableparsing technique must be sensitive to these semantic constraints, so that syllable boundaries are placed between morphemes. For example, the word passport should be divided into the syllables pass and port (maintaining each constituent word), rather than $p a$ and sport. There may also be other cases in which alternate syllable boundaries might be preferred, such as those discussed by Treiman and Zukowski (1990). Nevertheless, a large majority of words can be decomposed and represented as described in the main text.
} 
Table 2

Description and Illustration of Steps in PSIMETRICA for Generating Phonological Representations of the Words Placemats and Amount

\begin{tabular}{|c|c|c|c|}
\hline \multirow[b]{2}{*}{ Step } & \multirow[b]{2}{*}{ Procedure } & \multicolumn{2}{|c|}{ Words and representations } \\
\hline & & Placemats & Amount \\
\hline 1 & Determine constituent phonemes & /plesmæts/ & /omaunt/ \\
\hline 2 & Decompose word into syllables & /[ples] [mæts]/ & $/[ə][$ maunt]/ \\
\hline 3 & Decompose syllables into phoneme clusters & $/[(\mathrm{pl})(\mathrm{e})(\mathrm{s})][(\mathrm{m})(æ)(\mathrm{ts})] /$ & $/[(\varnothing)(\partial)(\varnothing)][(\mathrm{m})(\mathrm{au})(\mathrm{nt})] /$ \\
\hline 4 & Decompose phonemes into features & & \\
\hline
\end{tabular}

Note. $\quad$ PSIMETRICA $=$ Phonological Similarity Metric Analysis; $\varnothing=$ null phoneme.

by a given dimension. This step is essential for making the dissimilarity profiles valid indicators of how two words are related phonologically. If the phonemes in their component syllables are not properly aligned, then relevant similarities and differences between them cannot be taken fully into account. Also, because the phonological structure of the words is complex, procedures for aligning the words properly must be somewhat intricate.

In the next sections, we describe the alignment procedures for measuring three dimensions of the dissimilarity profile: the onset, nucleus, and coda dissimilarity. These dimensions are not exhaustive; other interesting ones (e.g., see Footnote 6) might also be considered. Furthermore, they are not unique: Different choices could be made about many of their definitional details; whether these choices matter for our purposes is primarily an empirical question. Nevertheless, the three dimensions of onset, nucleus, and coda dissimilarity that we have quantified here provide a reasonably complete basis on which to measure phonological dissimilarity between syllables and to predict serial recall accuracy precisely across different word sets.

Onset alignment. Onset phoneme clusters may contain between zero and three consonant phonemes. To align the onset clusters for a pair of syllables, we use a template related to structures discussed by Fudge (1969) and Hartley and Houghton (1996). This template has three positions or slots, each of which holds either an actual or a null phoneme. If a syllable onset contains the phonemes /s/, / $/$ (pronounced $s h$ ), /z/, or /3/ (pronounced $z h$ ), then it is put in the first template position. This is done because these phonemes are highly similar to each other and none of them can follow any other phonemes in the onsets of English syllables. Next, if the onset contains the phonemes /r/, /1/, $/ \mathrm{y} /$, or $/ \mathrm{w} /$, then it is placed in the third template position. This is done because each of these phonemes can follow phonemes other than those placed in the first template position and must immediately precede the vowel of a syllable. Any other onset phonemes are put in the middle position of the onset template. Remaining empty positions of the onset template are filled with null consonants $(\varnothing)$. This yields a uniform schema for representing the onset of any English syllable and allows corresponding onset phonemes to be aligned and compared directly.

Nucleus alignment. Nucleus phoneme clusters may contain either one or two vowel phonemes. When two nuclei with the same number of vowels are compared, we align them vowel by vowel. When a nucleus with one vowel is compared with a nucleus with two vowels, we double the vowel of the smaller nucleus, so that the doublets are aligned with the vowels in the larger nucleus.

Coda alignment. Coda phoneme clusters may contain between zero and five consonant phonemes, including two /s/s. Because of this potential complexity, it is difficult to use a single template with a fixed number of positions for aligning the phonemes of two codas. If such a template were used, the most appropriate template position for an /s/ would depend on the coda with which it is being compared.

For example, consider the codas of the following four words: mast (/mæst/), mass (/mæs/), mats (/mæts/), and masts (/mæsts/). A template must accommodate masts, and so must have two slots for /s/, before and after the slot for /t/. Clearly, the placement of the /s/ of mass depends on whether mass is being compared with mast or mats. When mass is compared with mast, the /s/ should be placed in the first /s/ slot, but when mass is compared with mats, the /s/ should be placed in the second /s/ slot to embody the similarities between these words.

To deal with these complexities, we take a different approach in our alignment procedure for codas than for onset clusters. When we align two coda clusters, we construct multiple candidate representations for each coda by adding null phonemes to the beginning and end of each cluster. We then find which two candidate representations are most similar, using a metric described later. This procedure yields representations that have the /s/ in mass aligned with both the /s/ in mast and the /s/ in mats.

Illustrative example. Our procedures for constructing the appropriate alignments between paired words for the onset, nucleus, and coda dimensions of the dissimilarity profile may be illustrated with the words amount and placemats. Table 3 shows the final alignments that would result for this pair of words. For the onset dimension, the onset phonemes are placed into templates. Here, the onset of the first syllable in placemats is aligned with three null phonemes in amount. The onsets of the second syllables are both represented as $1 \varnothing \mathrm{m} \varnothing /$ and aligned accordingly. For the nuclei of the first pair of syllables, the phonemes are aligned directly because each word contains a single vowel, /ə/ and /e/, respectively. In contrast, for the nuclei of the second pair of syllables, the /æ/ of placemats is doubled and then aligned with both the /a/ and the /U/ of amount. For the codas, the most similar pair of all possible comparisons is chosen. Because the coda of the first syllable in amount has no phonemes, a null phoneme is aligned with the /s/ in placemats. Null phonemes are added to the codas of the second syllables in each word, so that the /t/s are aligned directly, whereas the $/ \mathrm{s} /$ of placemats and the $/ \mathrm{n} /$ of amount are each aligned with a null phoneme.

This process yields an alignment of the phonemes in placemats and amount for each dimension of our dissimilarity profile, across both syllables of the two words. None of the word sets used in our experiments contains words with differing numbers of syllables, and so this technique is sufficient to obtain dissimilarity profiles 
Table 3

Alignment Used in PSIMETRICA for Phonological Representations of the Words Placemats and Amount

\begin{tabular}{|c|c|c|c|c|c|c|c|}
\hline \multirow[b]{3}{*}{ Word } & \multirow[b]{3}{*}{ Phonemic representation } & \multicolumn{6}{|c|}{ Syllable alignment } \\
\hline & & \multicolumn{3}{|c|}{ First } & \multicolumn{3}{|c|}{ Second } \\
\hline & & Onset & Nucleus & Coda & Onset & Nucleus & Coda \\
\hline $\begin{array}{l}\text { Placemats } \\
\text { Amount }\end{array}$ & $\begin{array}{c}/[(\mathrm{pl})(\mathrm{e})(\mathrm{s})][(\mathrm{m})(\mathfrak{x})(\mathrm{ts})] / \\
/[(\varnothing)(\mathrm{\partial})(\varnothing)][(\mathrm{m})(\mathrm{au})(\mathrm{nt})] /\end{array}$ & $\begin{array}{l}1 \varnothing \mathrm{p} \mathrm{1/} \\
1 \varnothing \varnothing \varnothing /\end{array}$ & $\begin{array}{l}\text { le/ } \\
/ \mathrm{a} /\end{array}$ & $\begin{array}{l}\text { /s/ } \\
1 \varnothing /\end{array}$ & $\begin{array}{l}1 \varnothing \mathrm{m} \varnothing / \\
1 \varnothing \mathrm{m} \varnothing /\end{array}$ & $\begin{array}{l}\text { /æ æ/ } \\
/ \mathrm{a} \mathrm{u} /\end{array}$ & $\begin{array}{l}1 \varnothing \mathrm{ts} / \\
/ \mathrm{nt} \varnothing /\end{array}$ \\
\hline
\end{tabular}

Note. $\quad$ PSIMETRICA $=$ Phonological Similarity Metric Analysis; $\varnothing=$ null phoneme

for these words. If paired words that have different numbers of syllables are compared, additional alignment procedures would be needed for each dimension of the dissimilarity profile.

\section{Obtaining a Dissimilarity Profile}

After the phonological representations for a pair of words have been aligned, PSIMETRICA's next step is to measure the phonological dissimilarity on each dimension of the dissimilarity profile. For present purposes, the measurement of phonological dissimilarity proceeds in substeps. It begins with calculations at the level of corresponding phonemes in the aligned representations of two words. Next, the mean dissimilarity between corresponding phoneme clusters and syllables is calculated. Then we calculate the overall mean dissimilarity between the words. Each of these substeps is described below.

Measurement of phonological dissimilarity between phonemes. In our phonological representations of words, each phoneme has a unique combination of binary phonological features. On the basis of these features, we measure the phonological dissimilarity between two corresponding phonemes in a pair of words by counting the number of mismatching features that the phonemes have and dividing it by the total number of relevant features per phoneme. ${ }^{8}$ This is analogous to measuring the distance between phonemes. Consequently, two identical phonemes would have a numerical dissimilarity of zero, whereas two extremely different phonemes would have a dissimilarity value closer to one.

There are also other special cases that must be accommodated in measuring phonological dissimilarity at the phonemic level. As mentioned already, our procedures for aligning the phonological representations of paired words frequently require consonant phonemes in syllable onset and coda clusters to be compared with corresponding null phonemes. Regarding these cases, we give them appropriate default dissimilarity values, depending on the phonemes' serial positions. For each distinct position in a syllable's onset, we calculate the mean dissimilarity between (a) the consonant phonemes that can legally fill this position and (b) the full set of consonant phonemes. This yields default dissimilarity values of $.36, .37$, and .53 for the three possible syllable-onset positions; comparisons between consonant and null phonemes in the first, second, and third serial positions of syllable onsets, respectively, are given these values. For comparisons between consonant and null phonemes in syllable codas, our dissimilaritymeasurement method involves an analogous calculation; on the basis of it, these comparisons are each given default dissimilarity values of 0.37 . The dissimilarity between a pair of null phonemes is given a value of 0 .

Calculation of the dissimilarity profile for a pair of words. After dissimilarity values have been calculated for each pair of phonemes identified by and aligned for every dimension of the dissimilarity profile, the mean values on these dimensions are obtained. This is first done for each pair of syllables by finding the average dissimilarity of the phoneme pairs of each dissimilarity dimension. Then, the mean value across syllables for each dimension of the dissimilarity profile is calculated, which produces the overall phonological dissimilarity profile for that word pair.

Calculation of the dissimilarity profile for a set of words. For an entire set of words, such as that which might be used to construct word sequences in the serial recall task, phonological dissimilarity is measured by averaging across the dissimilarity profiles of all possible word pairs from the set. In an $n$-word set, each word can be paired with $n-1$ other words, yielding $n(n-$ 1)/2 pairs. Averaging the dissimilarity values on each dimension for these pairs gives a mean dissimilarity profile for the word set as a whole.

Illustrative example. To illustrate how PSIMETRICA may be applied, we again consider the words placemats and amount (Table 4). For these words, the syllables, phoneme clusters, and phonemes first must be aligned in the manner explained previously (cf. Table 3). Next we compare the aligned phonemes in each pair and give them a dissimilarity value. When both of the phonemes of a pair are nonnull, this value equals the proportion of nonnull features that differ between the phonemes. These proportions appear as fractions in Table 4. For example, the phonemes /e/ and /ə/ have nine relevant features, and only two of these features are different. So, when /e/ and /ə/ are compared in the first syllables of placemats and amount, they are given a dissimilarity value of $2 / 9$. Pairs of phonemes that contain one actual phoneme and one null phoneme are given a default dissimilarity value that depends on the position of the phoneme pair. These default values appear as decimal numbers in Table 4. For example, in the codas of the first syllables of placemats and amount, the phonemes /s/ and / $\varnothing /$ are paired with each other, and because one of them is null, this phoneme pair is given a dissimilarity of 0.37 . Pairs of null pho-

\footnotetext{
${ }^{8}$ Most phonemes do not have values for all 13 features, because some features are specific to consonants and others to vowels. Consequently, the number of feature mismatches is divided by the number of features for which at least one phoneme has a value.
} 
Table 4

Illustration of Phonological Dissimilarity Measurement With PSIMETRICA for the Words Placemats and Amount

\begin{tabular}{|c|c|c|c|c|}
\hline \multirow[b]{2}{*}{ Phoneme clusters } & \multicolumn{2}{|c|}{ Word } & \multirow{2}{*}{$\begin{array}{c}\text { Phonological dissimilarity } \\
\text { of phonemes }\end{array}$} & \multirow{2}{*}{$\begin{array}{c}\text { Mean dissimilarity } \\
\text { of clusters }\end{array}$} \\
\hline & Placemats & Amount & & \\
\hline First onset & $1 \varnothing \mathrm{p} 1 /$ & $1 \varnothing \varnothing \varnothing 1$ & $-, 0.37,0.53$ & 0.45 \\
\hline First nucleus & le/ & $/ \partial /$ & $2 / 9$ & 0.22 \\
\hline First coda & $/ \mathrm{s} /$ & 101 & 0.37 & 0.37 \\
\hline Second onset & $1 \varnothing \mathrm{m} \varnothing /$ & $1 \varnothing \mathrm{m} \varnothing \mid$ & $-, 0 / 11,-$ & 0.00 \\
\hline Second nucleus & /æ æ/ & /a u/ & $1 / 9,4 / 9$ & 0.28 \\
\hline Second coda & $1 \varnothing \mathrm{t} \mathrm{s} /$ & $\operatorname{lnt} \varnothing /$ & $0.37, \% / 11,0.37$ & 0.25 \\
\hline
\end{tabular}

Note. Dashes indicate a comparison between null phonemes; $\varnothing=$ null phoneme. PSIMETRICA $=$ Phonological Similarity Metric Analysis.

nemes have no phonological features, and dissimilarity values are not given to them.

After dissimilarity values have been given to all of the aligned phoneme pairs of placemats and amount, a single mean value for each phoneme cluster is calculated by averaging across the dissimilarity values of the phoneme pairs within the cluster. Dissimilarity values for pairs of null phonemes are not included in this average. These mean dissimilarity values for the clusters of placemats and amount appear in Table 4. For example, the mean dissimilarity value of the second nuclei in placemats and amount is 0.28 . Finally, a dissimilarity profile is obtained by averaging the dissimilarity values of each phoneme cluster across syllables. For placemats and amount, this profile is $0.23,0.25$, and 0.31 (respectively, the mean dissimilarity values of the onsets, nuclei, and codas). By averaging such profiles from all possible pairs of words in a set, we obtain the word sets' overall mean dissimilarity profile.

\section{Relation to Previous Treatments of Phonological Coding and Similarity}

PSIMETRICA has some significant advantages over previous treatments of phonological similarity in research on VWM and serial recall (cf. Baddeley, 1986; Baddeley \& Andrade, 1994; Caplan \& Waters, 1994; Lovatt et al., 2000). Unlike measures of phonological similarity or dissimilarity obtained through subjective ratings, the ones obtained through PSIMETRICA have a formal theoretical basis: They are precise, reproducible, and sensitive to fundamental aspects of representation that presumably mediate phonological-similarity effects on performance of VWM tasks. PSIMETRICA assumes that phonological representations of words and their phonological features encode basic information relevant to the vocal production of speech sounds (Chomsky \& Halle, 1968). This assumption may be especially apt because according to the memory literature (e.g., Gupta \& MacWhinney, 1995; Murdock, 1974; Wickelgren, 1965, 1966), VWM typically has an articulatory rather than auditory (e.g., acoustic waveform or formant) nature.

In other respects, PSIMETRICA resembles methods used by Vitz and Winkler (1973) for measuring the judged dissimilarity of words and by Nairne (1990) for modeling immediate memory. Like them, we obtain numerical values between zero and one for each pair of elements being compared, with larger values corresponding to greater dissimilarity. Also related to PSIMETRICA is a method used by Frisch (1997), who measured similarity in terms of the number of natural classes that the phonological features of two phonemes share.

Nevertheless, PSIMETRICA makes some new contributions to the measurement and analysis of phonological dissimilarity that go significantly beyond previous attempts. To be specific, because different aspects of a word's phonetic structure may be important under different conditions, PSIMETRICA treats phonological dissimilarity as a multidimensional quantity. Also, PSIMETRICA's use of phonological features is more precise than that of Vitz and Winkler (1973), but our hierarchical representation allows the dissimilarity of entire words to be assessed and is consequently broader than Frisch's (1997) similarity metric, which compared only individual phonemes.

\section{A Method for Measuring Articulatory Duration}

Of course, just measuring phonological dissimilarity will not enable the phonological-loop model to be tested against other models of VWM. A valid method for measuring the articulatory durations of to-be-recalled items is also required. Such a method must be sensitive to the articulatory durations that are claimed by the phonological-loop model to cause the word-length effect. According to this model, the relevant durations are the actual times taken by participants to rapidly rehearse sequences of words. If the durations of articulatory rehearsal are not measured properly, then an experiment may yield misleading or irrelevant measurements for testing the phonological-loop model. Yet few (if any) previous experiments that attempted to test this model have measured articulatory durations in an ideal manner.

Given these considerations, in the next section, we review various methods that have been used previously for measuring articulatory durations, and we outline exactly what their deficiencies are. Next, we establish a set of relevant criteria that a valid method for measuring articulatory duration should satisfy. Then we describe a method that attempts to satisfy these criteria and that was used in our present experiments.

\section{Previous Methods for Measuring Articulatory Duration}

In previous experiments on VWM and serial recall, articulatory durations have been measured with a variety of methods. These methods fall into four general categories: (a) measurement of durations for isolated words, (b) measurement of durations for words in short, repeated, constant-length sequences, (c) measure- 
ment of durations for words read from lists, and (d) measurement of durations for words produced during final serial recall. Each of these methods has its own strengths and weaknesses, but because of their weaknesses, none of them is entirely sufficient for present purposes.

Measurement of articulatory durations for isolated words. Perhaps the simplest way to measure the articulatory duration for a word is to have a participant or a confederate pronounce the word aloud in isolation and record the time taken to do so. This method was used in several of Baddeley et al.'s (1975) original experiments. Also, it was the sole method used by Caplan et al. (1992), and it was a method used by Lovatt et al. (2000) to facilitate the initial selection of stimuli.

Some aspects of isolated-word duration measurement are relevant to the immediate serial recall task, because results from this method do provide some indication of whether one word is longer than another. However, measuring articulatory durations in this way also has serious deficiencies for testing the phonological-loop model. Because this method does not measure the durations of rapidly articulated sequences of words, which are the sine qua non of overt and covert articulatory rehearsal, the obtained measurements may be inadequate to test this model's predictions about duration effects on serial recall accuracy and memory span. For example, they may fail to take coarticulation effects on the duration of word sequences properly into account.

Measurement of articulatory durations for words in short, repeated, constant-length sequences. Another common method for measuring articulatory duration involves having participants rapidly repeat two or three words several (e.g., 10) times and recording the total times of their utterances. This method was used by Baddeley et al. (1975) in their Experiment V, by Baddeley and Andrade (1994) under both overt and covert articulation conditions, and by Lovatt et al. (2000). It is an improvement over isolated articulation, but it also has some weaknesses, because it fails to measure aspects of articulatory duration relevant to the phonological-loop model.

For example, in the immediate serial recall task, participants often have to rehearse and recall sequences that contain between five and seven words. The mental processes involved in planning and executing the articulation of such sequences certainly differ from the processes involved in articulating word pairs repeatedly. In addition, some factors that influence articulatory duration, such as interactions between tongue twister words, might occur only when specific combinations of words are articulated, and these combinations may not be present when limited sets of word pairs or triplets are involved. Consequently, experiments that use this method for measuring articulatory durations cannot adequately test the phonological-loop model.

Measurement of articulatory durations for words read from lists. A third method that has been used to measure articulatory duration is list reading. For this method, typical lists of words from the memory-span task are presented visually, and participants are instructed to read the lists aloud. The duration of their articulation is then measured. This method was used by Baddeley et al. (1975), Caplan and Waters (1994), Service (1998), and Lovatt et al. (2000).

One notable advantage of this method is that it involves word sequences like those used in the serial recall task. Consequently, the obtained articulatory durations are more relevant than some other measures. Nevertheless, this method has some serious defi- ciencies, too. For example, the lists of words are read, which is inadequate for testing the phonological-loop model because during the immediate serial recall task, words must be recalled from memory. In addition, list reading probably encourages participants to enunciate the words clearly and deliberately rather than to articulate them rapidly (as is presumably done during rapid memory-based rehearsal). Consequently, experiments that use the list-reading method may not test the phonological-loop model adequately.

Measurement of articulatory durations for words during final serial recall. A fourth method for measuring articulatory duration has been to record how long a participant takes to produce each sequence of words during final serial recall. This method was used by Service (1998) and Cowan et al. (1997), as well as in other experiments that are less directly relevant here (e.g., Cowan, 1992; Cowan et al., 1998; Dosher \& Ma, 1998; Schweickert et al., 1990). There are two major strengths of final recall duration measurement: It measures articulatory duration in vivo, while the participant is actually performing the serial recall task, and it measures an aspect of articulatory duration that the phonological-loop deems important.

However, this method also has some shortcomings. Most important, articulatory durations measured during final serial recall may not closely approximate those that occur during rehearsal, because recall might involve more memory search and reconstruction than does rehearsal. Given the phonological-loop model assumes that articulatory duration primarily affects rehearsal, this measurement method may not test the model adequately, either.

\section{A New Method for Measuring Articulatory Duration}

The preceding review suggests that a new method for measuring articulatory durations is needed to properly test the phonologicalloop model. Specifically, to measure the mean duration per word for a set of words, multiple (e.g., 15-20) sequences of several lengths (e.g., two to five words per sequence) should be constructed from each word set. On each duration-measurement trial, one sequence of words should be presented, and the participant should be allowed to study it until he or she has committed it to memory. When the participant is ready, the sequence should be articulated from memory, as presumably happens during motivated rehearsal. The sequence should be articulated at least twice, as may happen during iterative rehearsal, and the total time taken by the participant to articulate the words overtly should be measured. ${ }^{9}$ Participants should be encouraged to articulate the words rapidly and accurately. A duration-measurement trial should be repeated if a speech or memory error occurs during it. Finally, a mathematical analysis of total articulation times should be performed, and parameters of articulatory duration that are constant, linear, and

\footnotetext{
${ }^{9}$ The use of overt articulation is justified for at least three reasons: First, maximum overt and covert articulation rates are essentially the same (Baddeley \& Andrade, 1994; Landauer, 1962). Second, for overt articulation, the experimenter can monitor participants' speech errors, thereby enabling durations from aberrant trials to be assessed and discarded if necessary. Third, the moments at which articulation starts and stops can be identified more accurately when participants speak overtly.
} 
curvilinear with respect to sequence length should be estimated. ${ }^{10}$ As a result, mean articulatory durations that are more relevant than previous ones for testing the phonological-loop model may be estimated. The procedure and formulas we use for this estimation appear in Appendix B.

\section{Overview of Experiments}

This article reports two experiments in which we used our new methods of measuring articulatory duration and phonological dissimilarity to test the phonological-loop model versus other models of VWM. During both experiments, participants performed a standard version of the verbal serial recall task (Baddeley, 1986). For each experiment, sequences of words were constructed from word sets that differed in terms of their mean articulatory durations, phonological dissimilarity, and phonological complexity.

In Experiment 1, we show that mean articulatory durations and phonological dissimilarity account for an extremely large proportion of variance in memory-span data across six word sets. The results of Experiment 1 also reveal that phonological complexity per se may have no reliable effects on memory spans over and above those attributable to mean articulatory durations and phonological dissimilarity. Experiment 2 extends these results to include three additional sets of words. We show that mean memory spans for these three word sets can be accurately predicted a priori on the basis of parameter estimates from the results of Experiment 1. Taken overall, our findings are consistent with the phonologicalloop model and constitute strong evidence for the importance of phonological coding, repetitive time-consuming articulatory rehearsal, and trace decay in the serial recall task.

\section{Experiment 1}

The purpose of Experiment 1 was to replicate and extend two experiments by Caplan et al. (1992, Experiments 2 and 3). We believe that their experiments warrant closer inspection for three reasons. First, in Caplan et al., Baddeley and Andrade (1994), Caplan and Waters (1994), and Lovatt et al. (2002), articulatory durations were measured seven times (Table 1), yet none of these measurements were made under conditions that (according to the phonological-loop model) mediate the articulatory-duration effect on memory span. Second, in one experiment, Caplan et al. (Experiment 2) found that the articulatory-duration effect was reversed: Sequences of long words were recalled more accurately than sequences of short words. Although this reversal contradicts the phonological-loop model, Caplan et al.'s speech-planning hypothesis cannot explain it either, because the two word sets were equally complex and so should have yielded equal memory spans. Thus, this finding needs to be evaluated further. Third, Caplan and Waters attempted but failed to replicate other results from Caplan et al. (Experiment 3) that had shown no difference between serial recall accuracy when phonological complexity was equated but mean articulatory durations differed across word sets. Additional testing might provide more information about why this latter failure happened. To achieve these objectives, we measured phonological dissimilarities and articulatory durations of words with our new methods.

\section{Method}

Participants. The participants were 6 undergraduate students at the University of Michigan with normal perceptual, cognitive, and motor abilities. They were paid for their participation.

Apparatus. The experiment was conducted with a Pentium-class PC using our own special purpose software. Auditory stimuli were presented by means of headphones, and visual stimuli were presented on the computer's super video graphics array (SVGA) display. Performance was monitored by an experimenter who sat next to the participant and interacted with the computer to record the participants' responses.

Stimuli. Six different sets of words were used for constructing word sequences to test verbal serial recall (Appendix C). Sets 1 and 2 contained the long and short two-syllable words from Experiment 2 of Caplan et al. (1992). Sets 3 and 4 contained the difficult and easy one-syllable words from their Experiment 3. Sets 5 and 6 contained new short and long words that we selected to be low and approximately equal in concreteness and written frequency (Coltheart, 1981). ${ }^{11}$ We also attempted to maximize intraset dissimilarity in Word Sets 5 and 6 by choosing words that began with distinct letters and sounds. Table 5 shows relevant characteristics of the words in each of the six sets used here.

Measurement of phonological dissimilarity. For Experiment 1, preliminary data analysis revealed that only the dissimilarity of syllable onsets within each word set appeared to affect performance; the dissimilarity of syllable rhymes and stress did not. ${ }^{12}$ Because of this, we used PSIMETRICA's measure of onset dissimilarity as the present index of phonological dissimilarity for each pair of words. The phonological dissimilarity for an entire set of words was obtained by calculating the average pairwise onset dissimilarity for the words in the set.

It should be emphasized that none of the six word sets in Experiment 1 was selected to contain highly similar words. Thus, our present situation differs from previous experiments that have investigated the effects of phonological similarity on serial recall accuracy (e.g., Baddeley, 1966; Conrad \& Hull, 1964; Schweickert et al., 1990), in which results were compared for sets of highly similar and dissimilar words. In those experiments, variations of phonological dissimilarity were generally large and obvious, whereas in our Experiment 1, variations of phonological dissimilarity were subtle and perhaps undetectable through casual inspection.

Design. The participants were tested individually with three procedures: verbal serial recall, articulatory-duration measurement for isolated words, and articulatory-duration measurement for words in memorized sequences. Testing occurred during two sessions on separate days. During the first session, articulatory durations of words in memorized sequences were measured for Word Sets 1, 2, 5, and 6. During the second session, articulatory durations for isolated words in Word Sets 1-6 were measured, followed by measurement of articulatory durations for words in memorized sequences from Word Sets 3 and 4. After the articulatory-duration mea-

\footnotetext{
${ }^{10}$ Curvilinear parameters may be needed in measuring mean articulatory durations because Sternberg et al. (1978) found that production times for sequences of rapidly recalled words are a concave-upward function of sequence length.

${ }^{11}$ Both concreteness (ranging from 100 to 700 ) and written frequency (ranging from 0 to 70,000 based on the corpus of Kučera and Francis, 1967) were obtained from the MRC psycholinguistic database machine usable dictionary (Version 2.0; Wilson, 1987).

${ }^{12}$ There are various possible reasons why the contributions of syllable nuclei and codas to phonological-dissimilarity effects on performance were relatively unimportant here. For example, during rapid rehearsal, participants may have articulated mainly the onsets of syllables, which could lead them to be most important. However, different experimental procedures might induce other parts of the syllable to have more influence, so our present emphasis of syllable-onset dissimilarity might not generalize to all situations.
} 
Table 5

Characteristics of Word Sets 1-6 in Experiment 1

\begin{tabular}{|c|c|c|c|c|c|c|}
\hline \multirow[b]{2}{*}{ Variable and statistic } & \multicolumn{6}{|c|}{ Word set } \\
\hline & 1 & 2 & 3 & 4 & 5 & 6 \\
\hline \multicolumn{7}{|l|}{ Syllable numerosity } \\
\hline$M$ & 2 & 2 & 1 & 1 & 1 & 3 \\
\hline \multicolumn{7}{|l|}{ Phoneme numerosity } \\
\hline$M$ & 6.00 & 5.13 & 3.00 & 3.00 & 3.25 & 7.50 \\
\hline$S D$ & 0.70 & 0.30 & 0.00 & 0.00 & 0.60 & 1.30 \\
\hline \multicolumn{7}{|l|}{ Phonological dissimilarity } \\
\hline M & 0.401 & 0.344 & 0.266 & 0.336 & 0.399 & 0.363 \\
\hline$S D$ & 0.14 & 0.08 & 0.14 & 0.18 & 0.11 & 0.08 \\
\hline \multicolumn{7}{|l|}{ Written frequency } \\
\hline$M^{\mathrm{a}}$ & 16 & 16 & 30 & 45 & 18 & 17 \\
\hline$S D^{\mathrm{a}}$ & 16 & 16 & 27 & 67 & 16 & 23 \\
\hline \multicolumn{7}{|l|}{ Concreteness } \\
\hline$M$ & - & - & - & - & 304 & 296 \\
\hline$S D$ & - & - & - & - & 40 & 26 \\
\hline
\end{tabular}

Note. Dashes indicate that corresponding statistics regarding the concreteness of these words were not reported.

${ }^{a}$ Caplan et al. (1992) reported the means and standard deviations for the written frequency of Word Sets 1-4.

surements in the second session, participants performed a verbal serial recall task for each of the six sets of words. We randomized the order in which the word sets were used for each participant.

Measurement of articulatory durations for isolated words. To measure articulatory durations for isolated words, we had participants separately read each word from a set, speaking clearly and pausing before each word. The participants' utterances were recorded digitally. Their articulatory durations were measured later by identifying the beginning and end of the waveform for each word, using standard computer software.

Measurement of articulatory durations for words in memorized sequences. To measure the articulatory durations for words in memorized sequences, we used the method introduced earlier. With this method, a sequence of words was presented on the SVGA display in a horizontal row at the start of each trial. The word sequence remained on the SVGA display until the participant verbally indicated that he or she was ready to begin. Then, three 100-ms tones were presented at 500-ms intervals. Immediately after the third tone, the word sequence disappeared from the SVGA display and a timer began. As instructed, the participant then clearly articulated the sequence of words twice from memory at a moderately rapid pace, as if he or she was rehearsing the sequence for later serial recall. Trials on which the participant committed a speech error or failed to recall all of the words in a sequence were discarded and repeated for this sequence. When the participant completed the final word of a sequence, the experimenter pressed a key that stopped the digital timer. Then a new sequence of words was presented, and the process was repeated. ${ }^{13}$

Articulatory-duration measurements for each set were made in a single trial block. Before each block began, 13 three-word sequences, 10 fourword sequences, and 8 five-word sequences were constructed randomly with uniform probability so that no word occurred more than once per sequence, and all words occurred with approximately equal frequency throughout the block. Because Word Set 6 contained words that were considerably longer than those in the other sets, and because previous pilot participants had difficulty accurately repeating sequences of five words constructed from Word set 6 , the blocks in which articulatory durations were measured for Word sets 5 and 6 included 20 three-word sequences and 15 four-word sequences but no five-word sequences.

Measurement of serial memory spans. To measure participants' memory spans for each word set, we presented sequences of words constructed from each set in six serial recall trial blocks (one block per word set). The words of each sequence were constructed by randomly choosing words from a set without replacement.
At the beginning of each serial recall trial, the participant was informed about the length of the impending to-be-recalled word sequence. The participant then listened to a sequence of words presented over headphones, with 1.5-s intervals between onsets. A brief tone was presented $1.5 \mathrm{~s}$ after the final word, signaling that serial recall should begin. Participants recalled the words aloud and received credit for being correct only if an entire sequence was reproduced in its original serial order. After recall was complete, the computer provided feedback about whether the sequence was recalled correctly.

A staircase testing procedure, similar to the one of Schweickert et al. (1990), was used to select the length of each sequence in our task. Each block of trials began with a sequence of four words. If a sequence was recalled correctly, then the next presented sequence was one word longer; if a sequence was not recalled correctly, the next sequence was one word shorter. For each word set, this continued for a total of 16 sequences.

\section{Preliminary Data Analysis}

Before evaluating the results of Experiment 1 in detail, we conducted preliminary data analyses to obtain estimates of memory spans and articulatory durations for words in memorized sequences. These analyses yielded an estimate of memory span and mean articulatory duration for each participant for each of the experiment's six word sets.

Estimation of memory spans. Memory span was estimated to be the fractional sequence length at which a participant had a $50 \%$ chance of recalling a sequence of words correctly. This estimation involved fitting a generalized binomial linear-regression model with a logistic link function (McCullagh \& Nelder, 1989) for each participant/word-set combination. Memory spans-analogous to LD50, "the lethal dose for $50 \%$ of the cases" (McCullagh \&

\footnotetext{
${ }^{13}$ Subsequent supplementary data analysis revealed that the measures of mean articulatory duration obtained through this timing procedure were virtually the same as ones based on evaluation of digitized speech waveforms (see Appendix B and Footnote 18).
} 
Table 6

Results From Experiment 1

\begin{tabular}{|c|c|c|c|c|c|c|}
\hline \multirow[b]{2}{*}{ Variable } & \multicolumn{6}{|c|}{ Word set } \\
\hline & 1 & 2 & 3 & 4 & 5 & 6 \\
\hline \multicolumn{7}{|l|}{ Duration (ms) } \\
\hline Isolated words & 562 & 432 & 509 & 403 & 418 & 672 \\
\hline Words in memorized sequences & 254 & 275 & 257 & 201 & 245 & 405 \\
\hline \multicolumn{7}{|l|}{ Memory span (no. of words) } \\
\hline Observed & 6.22 & 5.78 & 5.50 & 6.05 & 6.27 & 5.09 \\
\hline Predicted & 6.19 & 5.77 & 5.45 & 6.14 & 6.23 & 5.12 \\
\hline Residual $^{\mathrm{a}}$ & 0.027 & 0.012 & 0.049 & -0.094 & 0.038 & -0.033 \\
\hline
\end{tabular}

Note. Predicted memory spans are based on parameter values in Table 7.

${ }^{\text {a }}$ Standard error of residual memory spans is 0.186 for Word Sets $1-6$.

Nelder, 1989 , p. 25)—were calculated from the best fitting parameters of these functions.

Estimation of mean articulatory durations for words in memorized sequences. We estimated the mean articulatory durations for words in memorized sequences by analyzing the total times taken to repeat particular sequences twice during the durationmeasurement trials. Details of this analysis appear in Appendix B. For each word set, the analysis involved fitting concave-upward functions of sequence length (Appendix B, Equation B1) to the mean total articulation times produced by each participant. As discussed in the Results section, the fits of the time functions were typically excellent. A quantitative combination of parameters associated with these functions (Appendix B, Equation B3) provided an estimate of the overall mean articulatory duration per word for each word set (see Table 6).

Also, in conjunction with this analysis, outliers among the total articulation times from the duration-measurement trials were deleted. We did this by excluding any such time that differed by more than 2.5 standard deviations from what the fitted concave-upward functions of sequence length (Appendix B, Equation B1) would have predicted. The outliers occurred about equally infrequently across the different participants, word sets, and sequence lengths; they constituted less than $1.6 \%$ of the total data set.

\section{Results}

Table 6 summarizes the mean values of articulatory duration and memory span from the six sets of words used in Experiment 1. Table 5 shows the corresponding mean values of phonological dissimilarity and phonological complexity for these word sets. We discuss each of these variables and how they are related next.

Phonological dissimilarity. According to our PSIMETRICA method for measuring phonological dissimilarity, the words in Word Set 1 were considerably more dissimilar from each other than were the words in Word Set 2 (see Table 5). This difference agrees with the subjective ratings of Baddeley and Andrade's (1994, Experiment 3) participants. However, the present dissimilarity measurements for Word Sets 1 and 2 disagree with Caplan and Waters's (1994) participants, who rated the two sets as having nearly identical levels of phonological dissimilarity.

Such disagreement also occurred for Word Sets 3 and 4. Measurements based on PSIMETRICA suggest that the words in Word Set 4 were considerably more dissimilar to each other than were the words in Word Set 3. However, participants in Caplan and
Waters's (1994) study rated these two word sets as having virtually identical levels of dissimilarity. Consequently, it appears that Caplan and Waters's procedures were not sensitive enough to detect measurable differences in phonological dissimilarity. Yet it remains to be seen whether these differences affected performance in the serial recall task.

Articulatory durations for isolated words. Across the six sets of words in Experiment 1, there were large differences between the mean durations of words when participants articulated each word in isolation. A within-subjects analysis of variance (ANOVA) revealed that differences between these durations were highly reliable, $F(5,25)=55.0, p<.01$. As shown in Table 6 , we found that Word Set 1 (long words) yielded reliably longer mean articulatory durations than did Word Set 2 (short words), mean difference $[M \pm S D]=130 \pm 20 \mathrm{~ms}, t(25)=6.50, p<.01$. This is analogous to what Caplan et al. (1992, Experiment 2) found with these two word sets (mean difference $=174 \mathrm{~ms}$ ). Furthermore, we found that Word Set 3 (difficult words) yielded reliably longer mean articulatory durations than did Word Set 4 (easy words), mean difference $=106 \pm 20 \mathrm{~ms}, t(25)=5.30, p<.01$. This is analogous to what Caplan et al. (Experiment 3) found with these two word sets (mean difference $=96 \mathrm{~ms}$ ).

Articulatory durations for words in memorized sequences. The total articulation times that participants on average took to produce memorized sequences of words during the duration measurement trials are shown in Figure 2 (solid circles) as a function of sequence length. Also shown here are the theoretical concaveupward functions whose parameters yielded the mean articulatory duration per word for each of the six word sets in Experiment 1, as described in Appendix B. The fit between these functions and the observed mean total articulation times was reasonably good (rootmean square error [RMSE] was $87 \mathrm{~ms}$, relative to total articulation times that typically exceeded $2 \mathrm{~s}) .{ }^{14}$

\footnotetext{
${ }^{14}$ Figure 2 shows both observed mean data and total articulation-time functions based on the mean parameter values of Equation B1 (Appendix B) for each word set and participant. To calculate the observed mean data and time functions, we first subtracted the obtained function intercepts from each observed mean total articulation time. The geometric mean (across participants) of the residual values was then calculated, and these values were then added to the arithmetic mean of the previously subtracted intercepts to produce the observed mean data points. For each word set,
} 

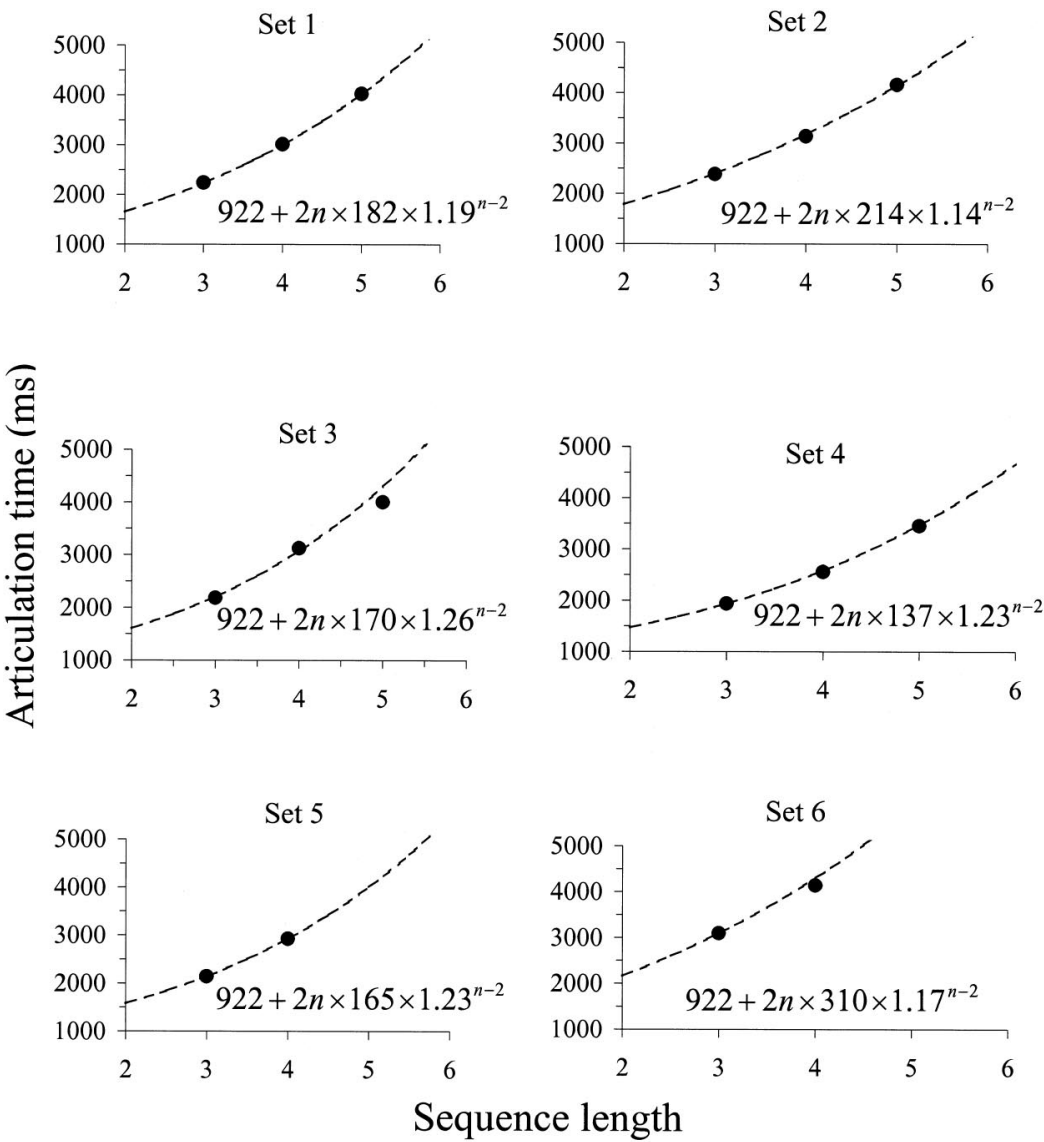

Figure 2. Observed mean total articulation times (solid circles) and theoretical total articulation-time functions (dashed lines) for each word set and sequence length in Experiment 1.

Table 6 shows the estimated mean articulatory duration per word for each word set. We found that the words articulated by participants in memorized sequences had durations whose means differed reliably across these sets, $F(5,25)=66.1, p<.01$. For example, under these conditions, the difference between the mean articulatory durations of the words in Word Set 5 and Word Set 6 was $160 \pm 12 \mathrm{~ms}, t(25)=13.30, p<.01$.

However, when participants articulated words in memorized sequences, their mean durations were considerably less than those obtained for isolated words (mean difference $=226 \pm 37 \mathrm{~ms}$ ), $t(5)=6.10, p<.05$ (see Table 6). Also, the rank orders of the mean articulatory durations across word sets differed reliably, depending on how the durations were measured, $F(5,25)=13.3$, $p<.01$. For example, when we measured the durations of the nominally short words of Word Set 2 by having participants articulate them in memorized sequences, their mean duration was actually a bit longer than the corresponding mean for the nominally long words of Word Set 1 (275 ms vs. $254 \mathrm{~ms}$ ), whereas Word Set 2 words had a much shorter mean duration than did

parameters of the displayed time function were obtained by calculating the arithmetic mean of the $I$ values and the geometric mean of the $a$ and $d$ values across participants. This type of averaging maintains the form of Equation B1 for the mean data.
Word Set 1 words when they were articulated in isolation (432 ms vs. $562 \mathrm{~ms})$. The interaction contrast between these mean duration differences $(151 \pm 23 \mathrm{~ms})$ was highly reliable, $t(25)=6.57, p<$ .01 .

As a result, our mean articulatory durations for words in memorized sequences differed reliably from the articulatory durations reported by Caplan and Waters (1994), who measured them by having participants read words in lists that were visible throughout the articulation period. With their procedure, Caplan and Waters found that Word Set 1 words were considerably longer than Word Set 2 words (531 ms vs. $477 \mathrm{~ms}$ ). In contrast, with our sequencerecall method, we found that the mean articulatory durations of these sets reversed their ordering (254 ms vs. $275 \mathrm{~ms}$ ). Such a reversal suggests that list reading may not yield entirely adequate measurements of articulatory durations for testing the phonological-loop model.

Memory spans. Across the six word sets in Experiment 1, participants' memory spans depended reliably on which set was used for constructing the to-be-recalled word sequences, $F(5$, $25)=6.1, p<.005$. For example, on average, the words of Word Set 4 yielded a reliably greater memory span than did the words of Word Set 3 (mean difference $=0.55 \pm 0.26$ words), $t(25)=2.12$, $p<.05$. This difference is similar to what Caplan and Waters (1994) found for these word sets; an analogous difference was also found by Caplan et al. (1992). On average, our participants also 
had marginally greater memory spans for the words of Word Set 1 than for the words of Word Set $2(M$ difference $=0.44 \pm 0.26$ words), $t(25)=1.69, p<.10$. Again this difference is analogous to what Caplan and Waters found.

It therefore appears that considerable agreement exists between our memory-span data and those of some previous investigators who have criticized the phonological-loop model. What we disagree about is the extent to which the systematic variance of mean memory spans across word sets can be explained by their correlations with mean articulatory durations and phonological dissimilarity, which are two principal predictor variables that should account well for memory spans if the phonological-loop model is veridical. Thus, to help resolve this disagreement, we performed the following additional analyses.

Correlations between memory spans, articulatory durations, phonological dissimilarity, and phonological complexity. Using the results from the six word sets of Experiment 1 (see Table 6), we performed a multiple regression analysis with the mean articulatory durations and phonological dissimilarities of words in memorized sequences as the predictor variables and the mean memory spans as the predicted variable. Across Word Sets 1-6, this analysis accounted for an extremely high and reliable percentage of variance in the memory-span data $\left(R^{2}=.986\right.$, adjusted $\left.R^{2}=.977\right)$, $F(2,3)=106.0, p<.002$. The RMSE between the observed and predicted mean memory spans in this analysis was extremely small (RMSE $=0.05$ words). The estimated regression coefficients that yielded this excellent fit appear in Table 7. Both of the predictor variables contributed reliably to the overall goodness of fit: For the mean articulatory durations (partial $r=-.99$ ), $t(3)=-12.60, p<$ .01 ; for the mean phonological dissimilarities (partial $r=.98$ ), $t(3)=8.50, p<.05$. Thus, if either articulatory duration or phonological dissimilarity had been omitted as a predictor variable in this regression analysis, then the fit to the observed memory spans would have been significantly poorer (Figure 3 ). ${ }^{15}$

After the estimated contributions of these predictor variables to memory spans were removed, there was no reliable correlation between memory-span residuals and phonological complexity $(r=$ $-.06), t(4)=-0.13, p>.05$. Furthermore, the residual span values were all very small and fell within confidence intervals that surrounded a null span value (see Table 6). These results are what would be expected if the phonological-loop model is basically correct about the nature of the mechanisms that mediate memory spans, whereas a model based on the speech-planning hypothesis of Caplan et al. (1992) cannot account for what we found.

To further test whether phonological complexity might be a significant predictor of memory spans, as Caplan et al.'s (1992) hypothesis implies, we conducted two more multiple regression analyses. In one of these, mean memory spans were the predicted variable, and there were three predictor variables: mean phonolog-

Table 7

Coefficients of Multiple Linear Regression Analysis for Predicting Memory Span Based on Articulatory Duration and Phonological Dissimilarity in Experiment 1

\begin{tabular}{lcl}
\hline \multicolumn{1}{c}{ Predictor } & Coefficient & \multicolumn{1}{c}{$S E$} \\
\hline Intercept (no. of words) & 5.50 & 0.24 \\
Articulatory duration (ms) & -0.00573 & 0.00045 \\
Phonological dissimilarity & 5.35 & 0.63 \\
\hline
\end{tabular}

ical complexity, phonological dissimilarity, and articulatory durations of words in memorized sequences. Across Word Sets 1-6 of Experiment 1, this analysis accounted for virtually the same percentage of variance in the memory-span data as did the preceding analysis that was based on only articulatory duration and phonological dissimilarity $\left(R^{2}=.986\right.$, adjusted $\left.R^{2}=.966\right), F(3,2)=$ $48.1, p<.05$. Including phonological complexity as a predictor variable did not reliably improve the goodness of fit between the observed and predicted mean memory spans, $F(1,2)=0.04, p>$ .50 . The correlation between the mean memory spans and phonological complexity was low and unreliable (partial $r=-.14$ ), $t(2)=-0.20, p>.50$.

We also conducted a complementary multiple regression analysis that included phonological complexity and phonological dissimilarity as predictor variables but excluded articulatory duration. This analysis, compared with the preceding ones, accounted for substantially less systematic variance in the memory-span data across the six word sets $\left(R^{2}=.816\right.$, adjusted $\left.R^{2}=.693\right)$. The fit between the observed and predicted memory spans obtained from this latter analysis was reliably worse than the fit obtained when articulatory duration was included as a predictor variable, $F(1$, $2)=24.9, p<.05$. These results further suggest that articulatory duration is crucial in accounting for memory spans, and if the contributions of this predictor variable are taken properly into account, then phonological complexity may be irrelevant.

Nevertheless, there is still another conceivable hypothesis that needs to be considered here (N. Cowan, personal communication, September, 2001). According to it, both memory span and mean articulatory duration of words in memorized sequences are byproducts of a single more basic underlying factor: item memorability. Perhaps such memorability determines the rate at which items are reproduced from subspan sequences as well as the probability of correct recall from supraspan sequences. If so, then our results would not support the phonological-loop model per se. Rather than showing that mean articulatory duration is a causal determinant of memory span as this model implies, the correlation that we found between these two variables might manifest some other mechanism whose operation is really responsible for memorability.

Yet, on balance, our results provide evidence against the latter memorability hypothesis. For example, it predicts that mean articulatory durations should be shorter in sequences of words with high phonological dissimilarity, because high phonological dissimilarity presumably increases their memorability. However, we found no support for this prediction; the correlation between phonological dissimilarity and articulatory duration was only .09 in Experiment 1.

\footnotetext{
${ }^{15}$ For example, Figure 3A reveals that the mean memory span for Word Set 3 was markedly overpredicted by the linear predictor coefficient associated with articulatory duration. Such overprediction occurred because this predictor coefficient, by itself, neglects to take account of phonological dissimilarity, which was especially low for Word Set 3 . Conversely, the mean memory span for Word Set 6 was markedly overpredicted by the predictor coefficient associated with phonological dissimilarity (Figure 3B). Such overprediction occurred because this predictor coefficient, by itself, neglects to take account of articulatory duration, which was especially long for Word Set 6 . Only by taking into account the contributions by both articulatory duration and phonological dissimilarity can mean memory spans be predicted with uniformly high accuracy.
} 

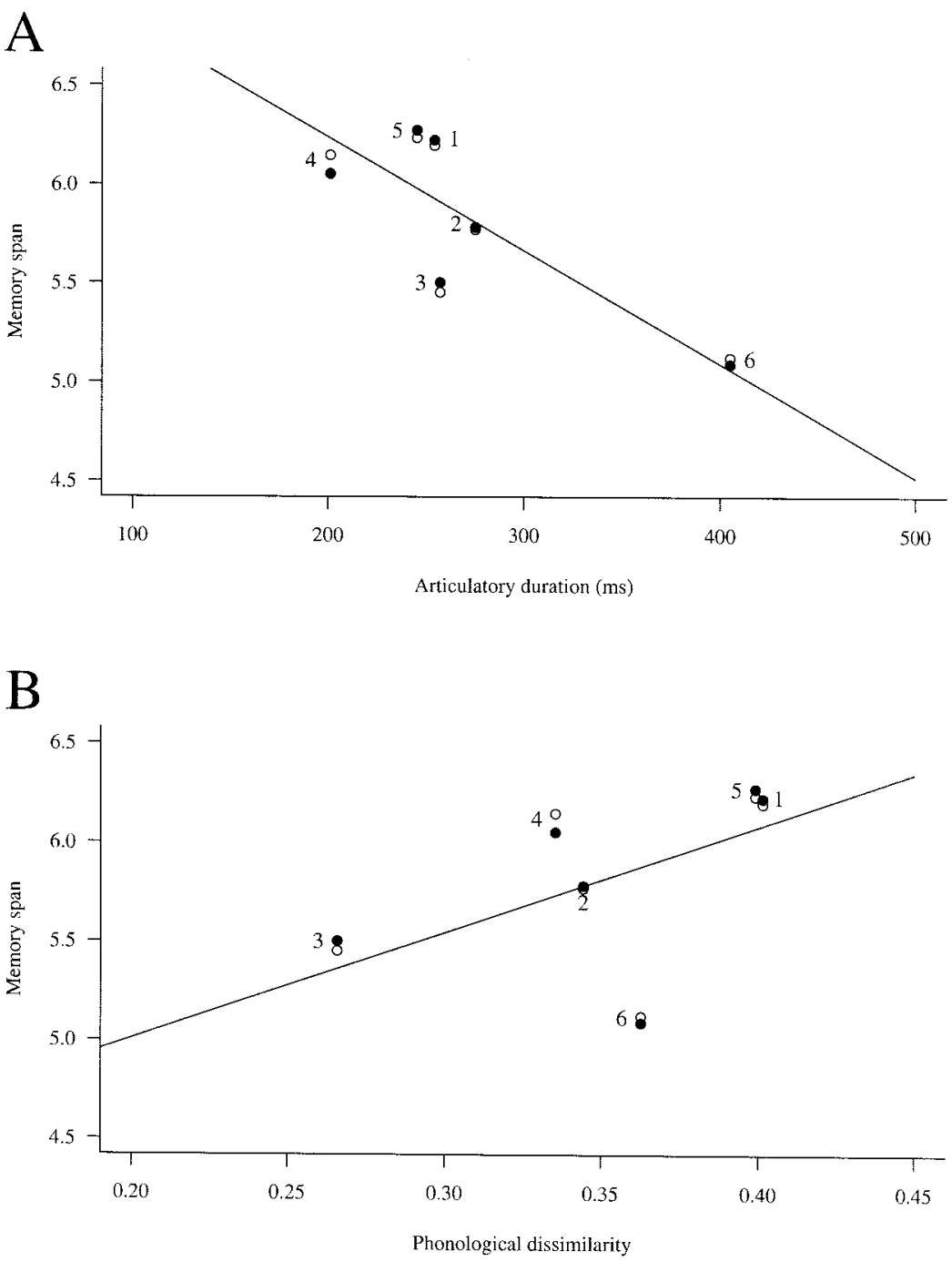

Figure 3. Mean memory span for each word set of Experiment 1. Lines show the estimated effect of each respective predictor variable on memory span, as determined from the linear regression coefficients in Table 7. Solid circles represent observed mean values; open circles represent corresponding predicted mean values when both predictor variables are taken into account. The numbers beside these symbols indicate the word sets from which they came. A: Mean articulatory duration for words in memorized sequences. B: Phonological dissimilarity.

Furthermore, this evidence against the memorability hypothesis is bolstered by two other facts. First, during our articulatoryduration measurement procedure, trials on which participants hesitated or made recall errors were discarded. Consequently, all measured articulation times came from cases in which the participants recalled the word sequences with relative ease. Second, the correlation between our two types of articulatory-duration measurement (memorized lists vs. isolated speech) was .84. This high correlation indicates that both of these measurements manifested similar processes, even though one imposed essentially no memory load, whereas the other required some memory. ${ }^{16}$

\section{Discussion}

Taken all together, the results of Experiment 1 strongly support the phonological-loop model and its predictions about perfor- mance of the serial recall task. We found that articulatory duration and phonological dissimilarity can account for nearly all of the variance in mean memory spans across the present six word sets,

\footnotetext{
${ }^{16}$ Evidence against this hypothesis can also be found in experiments reported elsewhere. For example, Cowan et al. (1998, Experiment 1) presented children with identical short sequences of numbers on six consecutive trials that the children then read rapidly. Audio recordings of the responses were made so that the durations of each word and the durations of the interword pauses could be measured precisely. Results showed that total articulation times decreased across the sequence of six trials, but the durations of the words were uncorrelated with the durations of the pauses between the words. If the durations of interword pauses are indications of retrieval speed, this demonstrates that a factor affecting memorability is unrelated to a direct measure of articulatory duration. We are grateful to $\mathrm{N}$. Cowan for proposing this explanation to us.
} 
including several used by previous investigators (Baddeley \& Andrade, 1994; Caplan et al., 1992; Caplan \& Waters, 1994). In contrast, phonological complexity offers less predictive power in Experiment 1. Our results yielded no evidence that the word-length effect stems from phonological complexity per se, so there is no reason to believe that rehearsal during the serial recall task is based solely on speech planning, as hypothesized by Caplan et al. (1992). Instead, the original assumptions of the phonological-loop model still appear to be veridical, and we conclude that rehearsal requires overt or covert articulation of word sequences whose duration affects memory spans significantly.

Experiment 1 also demonstrates that different procedures for measuring articulatory durations can yield values that differ in both absolute and relative magnitudes. When we measured the articulatory durations for words in memorized sequences constructed from Word Sets 1 and 2, Caplan et al.'s (1992) set of nominal long words yielded durations that were actually shorter than those for the matched set of nominal short words. There are several reasons why this may have occurred. First, our measurement method involved articulation of words from memory, whereas previous methods have not (cf. Caplan et al., 1992; Caplan \& Waters, 1994). Second, our instructions encouraged participants to articulate the words as if they were rehearsing and required repeating each memorized sequence of words twice. Consequently, this method created a situation that closely resembles rehearsal in the verbal serial recall task, so participants might have been less prone to unnecessarily extend their pronunciations of words. Third, we measured articulatory durations for several different sequence lengths. These measurements revealed that articulatory durations of words may differ in terms of both a baseline duration and a sequence-length amplification factor. As a result, an apparent duration difference between words for one sequence length may not generalize to differences for other sequence lengths. Thus, it is essential that these differences be taken into account when attempting to predict memory-span data accurately.

Given the results of Experiment 1, it can be seen likewise that PSIMETRICA is apparently more informative than some previous methods of measuring phonological similarity, such as those based on subjective ratings (cf. Baddeley \& Andrade, 1994; Caplan \& Waters, 1994). With phonological-dissimilarity measurements from PSIMETRICA, we successfully predicted reliable differences between the mean memory spans for Word Set 1 versus Word Set 2 and for Word Set 3 versus Word Set 4 (see Figure 3B). These predictions succeeded even though Caplan and Waters (1994) claimed, on the basis of subjective ratings, that Word Sets 1 and 2 had about equal degrees of phonological dissimilarity and that Word Sets 3 and 4 did as well.

The present success of PSIMETRICA presumably stems from it taking account of detailed matches and mismatches at the level of individual phonological features, whereas subjective ratings of phonological dissimilarity perhaps do not (cf. Vitz \& Winkler, 1973). We have been able to predict consistent effects of phonological dissimilarity on memory spans for sets of words that do not obviously differ in phonological dissimilarity, which is possible only because we characterize phonological dissimilarity at the phonological-feature level. Indeed, it appears that combinations of individual phonological features may be crucial for coding and storing phonemic information about words during serial recall and other VWM tasks (cf. Baddeley, 1986, 1992).
In summary, we conclude that the data of Caplan et al. (1992), who mounted one of the most influential critiques against the phonological-loop model, do not really justify rejecting this model's assumptions. The apparent inconsistencies between the predictions of the phonological-loop model and the data of Caplan et al. were probably obtained only because they measured phonological dissimilarity and articulatory duration in less than ideal ways. If, instead, these variables are measured through our methods, the strong correlations obtained between them and memory spans support the phonological-loop model.

\section{Experiment 2}

A principal objective of Experiment 2 was to generalize the results of Experiment 1. Our approach involved measuring participants' memory spans for word sequences constructed from three new sets of words across which mean articulatory durations and phonological complexity varied in a quasi-orthogonal manner. For two of these word sets, their phonological complexity was held constant, but the mean articulatory durations of their words differed significantly between sets. This aspect of Experiment 2 is analogous to the experimental designs of Baddeley et al. (1975, Experiment 4), Caplan et al. (1992), Caplan and Waters (1994), Service (1998), and Lovatt et al. (2000). However, unlike some of these investigators, we show that differences in mean articulatory durations account for large reliable amounts of variance in memory spans even when phonological complexity is constant across word sets. Furthermore, for two of the word sets in Experiment 2, phonological complexity differed significantly, but the mean articulatory durations of their words were approximately equal. This aspect of Experiment 2 is analogous to the experimental designs of Cowan et al. (1997) and Service. Unlike these investigators, however, we show that differences in phonological complexity fail to account for large reliable amounts of variance in memory spans when mean articulatory durations are equated across word sets. Taken together, such results are like what Experiment 1 yielded and what the phonological-loop model predicts, whereas they conflict with the predictions of some alternative models (e.g., the speech-planning hypothesis; Caplan et al., 1992).

Two crucial methodological innovations, which have been advocated and adopted already in this article, enabled us in Experiment 2 to generalize our results from Experiment 1. As during Experiment 1, we measured articulatory durations for words in recently memorized sequences with variable lengths. Also, to quantify the effects of phonological dissimilarity, we measured it on the basis of PSIMETRICA.

In addition, there was another important aspect of Experiment 2. As part of analyzing its results, the effects of both phonological dissimilarity and articulatory duration were quantified by the values of the multiple regression predictor coefficients that fit the memory spans from Experiment 1 . Thus, with Experiment 2, we show that the effects of these factors on memory spans may be essentially invariant across different groups of participants and experimental stimuli.

\section{Method}

Participants. The participants were 12 undergraduate students from the University of Michigan. None had participated previously.

Apparatus. The apparatus was the same as in Experiment 1. 
Table 8

Means and Standard Deviations of Variables for Word Sets in Experiment 2

\begin{tabular}{|c|c|c|c|c|c|c|}
\hline \multirow[b]{3}{*}{ Variable } & \multicolumn{6}{|c|}{ Word set } \\
\hline & \multicolumn{2}{|c|}{7} & \multicolumn{2}{|c|}{8} & \multicolumn{2}{|c|}{9} \\
\hline & $M$ & $S D$ & $M$ & $S D$ & $M$ & $S D$ \\
\hline Syllable numerosity & 2 & 0 & 2 & 0 & 3 & 0 \\
\hline Phoneme numerosity & 7 & 0 & 7 & 0 & 8 & 0 \\
\hline Phonological dissimilarity & 0.344 & 0.087 & 0.389 & 0.077 & 0.324 & 0.096 \\
\hline Familiarity & 489 & 99 & 464 & 62 & 485 & 73 \\
\hline Concreteness & 387 & 93 & 453 & 112 & 397 & 129 \\
\hline
\end{tabular}

Stimuli. To construct sequences of words for the serial recall task, we used three new word sets: Word Set 7, which contained short two-syllable words; Word Set 8, which contained long two-syllable words; and Word Set 9, which contained complex three-syllable words (see Appendix C). The words of Word Sets 8 and 9 were selected to have approximately equal mean articulatory durations even though Word Set 8 had fewer syllables and phonemes per word than did Word Set 9. The words of Word Set 7 were selected to have significantly shorter mean articulatory durations than those of Word Sets 8 and 9. However, the words of Word Sets 7 and 8 were equal in phonological complexity (i.e., two syllables and seven phonemes per word), whereas the words of Word Set 9 were significantly more complex (i.e., three syllables and eight phonemes per word). All three sets of words were approximately equal in familiarity and concreteness. ${ }^{17}$ Table 8 shows the mean values (and standard deviations) that each word set had for concreteness, familiarity, phonological complexity (indexed by syllable and phoneme numerosity), and phonological dissimilarity.

Given the several constraints imposed on these word sets, we could not perfectly equate the mean phonological dissimilarity of the words in each set with the mean phonological dissimilarity of the words in the other sets (see Table 8). Consequently, there was a partial confounding among phonological dissimilarity, phonological complexity, and articulatory durations across Word Sets 7-9. Nevertheless, by taking previous results from Experiment 1 into account, we separated the contributions of these predictor variables to the memory spans in Experiment 2.

Design. Each participant was tested individually in a single 1.5-hr session. After an introductory instruction period, each session included two phases of testing. In the first phase, the participant's mean articulatory durations were measured for the words in Word Sets 7, 8, and 9, respectively. In the second phase, the participant's memory spans were measured for sequences of words constructed from each set. The order in which the word sets were used during each phase was counterbalanced across participants, but each participant received the sets in the same order during both phases. Participants were paid $\$ 8$ plus bonuses for good performance on the serial recall task.

Articulatory-duration measurement. The articulatory durations for words in memorized sequences were measured as in Experiment 1. Each participant received sequences ranging from two to five words and performed eight measurement trials per length for each word set.

Memory-span measurement. For each word set, memory spans were measured as in Experiment 1. Each memory-span trial block included 20 trials. To encourage good performance, whenever the memory span for a word set exceeded four words, we paid the participant a bonus of $S-4$ dollars, where $S$ was the magnitude of the memory span.

Preliminary data analysis. One atypical participant correctly recalled sequences that included as many as 10 words. Although an articulatoryduration effect occurred for him, his memory spans far exceeded those of the other 11 participants, and he reported using a unique mnemonic strategy to achieve this performance. We therefore omitted his data from subsequent analyses, because they were clearly unusual and would have distorted the remaining group means. For all other participants, mean articulatory durations and memory spans were calculated and analyzed as in Experiment 1.

\section{Results}

Table 9 summarizes the mean values of articulatory duration and memory span that resulted from the three sets of words in Experiment 2. Here we discuss our results with respect to each of these variables and the relationships among them compared with those from Experiment 1 (cf. Table 6).

Articulatory durations. As before, when participants articulated recently memorized sequences of words, their total articulation times increased in a concave-upward trend with sequence length. Figure 4 shows how well Equation B1 of Appendix B fit these times on average for each word set of Experiment 2 (RMSE $=19 \mathrm{~ms}$ ). On the basis of estimates obtained from parameters of this equation, the means of the articulatory durations per word differed reliably across word sets, $F(2,20)=28.6, p<$ .01. In particular, the difference between the mean articulatory durations for the long two-syllable words of Word Set 8 and the short two-syllable words of Word Set 7 was large and reliable (see Table 9) (mean difference $=58 \pm 8 \mathrm{~ms}), t(20)=7.25, p<.01$. This result confirms that we succeeded in constructing two sets of words across which the mean articulatory duration per word differed even though their degrees of phonological complexity (i.e., numbers of syllables and phonemes per word) were equated. Moreover, the difference between the mean articulatory durations for the long two-syllable words of Word Set 8 and the complex three-syllable words of Word Set 9 was small and unreliable (Table 9) (mean difference $=7 \pm 8 \mathrm{~ms}$ ), $t(20)=0.87, p>.25$. This result confirms that we succeeded in constructing two sets of words across which the mean articulatory durations per word were nearly equal despite there being a marked difference in their phonological complexity. Thus, in Experiment 2, mean articulatory durations and phonological complexity varied quasiorthogonally, which enables us to separate the effects of these predictor variables on memory spans. ${ }^{18}$

${ }^{17}$ Both concreteness and familiarity measures range from 100 to 700 , as reported by the MRC psycholinguistic database machine usable dictionary (Version 2.0.; Wilson, 1987).

${ }^{18}$ Our mathematical analysis was designed to remove any biases contributed by the experimenter to our measurement of participants' mean word durations in articulating sequences from memory. To verify that this analysis succeeded, we have reexamined the data from 4 participants whose performance during Experiment 2 was tape recorded. Their utterances from articulating words in memorized sequences were evaluated with Audacity digital-waveform analysis software Version 0.97 (Mazzoni, 2001) to obtain new estimates of the mean articulatory duration for each 
Table 9

Results From Experiment 2

\begin{tabular}{lccc}
\hline & \multicolumn{3}{c}{ Word set } \\
\cline { 2 - 4 } \multicolumn{1}{c}{ Variable } & 7 & 8 & 9 \\
\hline Mean articulary duration (ms) & 393 & 451 & 444 \\
$\begin{array}{l}\text { Memory span (no. of words) } \\
\text { Observed }\end{array}$ & 5.21 & 5.05 & 4.71 \\
$\begin{array}{l}\text { Predicted } \\
\text { Residual }\end{array}$ & 5.09 & 5.00 & 4.69 \\
\hline
\end{tabular}

Note. Predicted memory spans are based on the same predictor coefficients for articulatory duration and phonological dissimilarity as in Experiment 1 (see Table 7).

${ }^{\text {a }}$ Standard error of residual memory spans is 0.099 for Word Sets 7-9.

Memory spans. Participants' memory spans (see Table 9) reliably depended on which word set was used for constructing the to-be-recalled sequences of Experiment 2, $F(2,20)=6.6, p<.01$. For example, on average, the short two-syllable words of Word Set 7 yielded a reliably larger memory span than did the complex three-syllable words of Word Set 9 (mean difference $=0.50 \pm$ 0.14 words), $t(20)=3.52, p<.001$. The mean memory span for the long two-syllable words of Word Set 8 was intermediate.

Correlations between memory spans, articulatory durations, phonological dissimilarity, and phonological complexity. To discover which predictor variables best accounted for the mean memory spans across the three word sets of Experiment 2, we used the same regression equation that had accounted well for memory spans from Experiment 1. According to this equation, $S=5.50-$ $0.00573 \times D+5.35 \times P$, where $S$ is the predicted mean memory span for sequences constructed from a particular word set, $D$ is the mean articulatory duration (in milliseconds) of the words in the set, and $P$ is their mean phonological dissimilarity; the numerical coefficients have been estimated with the results of Experiment 1 (cf. Table 7). Substituting the values of $D$ and $P$ for the three new word sets of Experiment 2 in the regression equation yielded the predicted mean memory spans in Table 9 , which may be compared with the observed mean memory spans for these sets.

From this comparison, we see that the predicted mean memory spans based on the regression equation fit the observed mean memory spans closely $\left(R^{2}=.991 ; \mathrm{RMSE}=0.076\right.$ words $)$. The residual differences between the observed and predicted mean memory spans were not reliable, $F(2,20)=0.3, p>.05$, indicating that the regression equation accounted for essentially all of the systematic variance in the observed mean memory spans across the three word sets of Experiment 2. The difference between the grand means of the observed and predicted memory spans was not reliable either, $F(1,10)=0.1, p>.50$, indicating that the regression equation accounted not only for the word-set effects on

word set and each participant. Results showed that mean estimated articulatory durations from the original measurements differed on average by $10 \mathrm{~ms}$ (ranging from 0 to $20 \mathrm{~ms}$ ) compared with those from the waveform analysis. Furthermore, these estimates were essentially unbiased (the mean difference between original and verification measurements equaled $0.4 \mathrm{~ms}$ across word sets and participants). observed memory spans but also for the memory spans' overall absolute magnitude.

This excellent fit was achieved even though Experiment 2 involved new sets of words and participants, but no new parameter values were estimated from its results (i.e., the fit provided by the regression equation is based solely on predictor coefficients estimated from the results of Experiment 1). The pairs of observed and predicted mean memory spans in Experiment 2 fit almost seamlessly with those that Experiment 1 yielded (see Figure 5). Thus, because the regression equation is based on articulatory durations and phonological dissimilarity rather than phonological complexity, its successful a priori prediction of the mean memory spans in Experiment 2 further supports the phonological-loop model of verbal serial recall.

On the other hand, the results of Experiment 2-again, like the results of Experiment 1-provide relatively little support for alternative models under which phonological complexity is claimed to be a powerful predictor of memory span (e.g., Caplan et al., 1992; Caplan \& Waters, 1994; Cowan et al., 1997). After the expected contributions of the mean articulatory durations and phonological dissimilarity were removed from the observed mean memory spans through the regression equation, the residual memory spans (see Table 9) revealed no reliable effect of phonological complexity, $F(2,20)=0.3, p>.50$. The residual memory spans were all very small and fell within confidence intervals that surrounded a null value. In particular, the residual memory span for the complex three-syllable words of Word Set 9 was neither less than zero nor reliably less than the residual memory span for the long two-syllable words of Word Set $8, t(10)=0.25, p>.50$, contrary to what Caplan et al. (1992; Caplan \& Waters, 1994) would have expected. That the complex words of Word Set 9 yielded the smallest observed mean memory span (see Figure 5, triangle labeled with 9) appears to have resulted simply because their articulatory durations were longer than those in Word Set 7 and their phonological dissimilarities were less than those in Word Set 8 .

\section{Discussion}

The results of Experiment 2 bolster the results of Experiment 1, confirming that phonological complexity is not a significant predictor of verbal serial memory span when the effects of articulatory duration and phonological dissimilarity have been taken properly into account. If complexity reduced (or enhanced) memory span independent of these other factor effects, then memory span for the complex three-syllable words of Word Set 9 in Experiment 2 should have been considerably less (or more) than we found. Although memory spans for sequences of words may depend on many factors, phonological complexity does not seem to matter much when phonological dissimilarity and articulatory duration are measured through our methods and then used in predicting performance. This is exactly what the phonological-loop model implies.

The results of Experiment 2 also provide further empirical justification for the methods that we have introduced to measure articulatory duration and phonological dissimilarity. When measured as we have described, these variables enable highly accurate accounts of memory span that generalize readily to new participants and different sets of words. If our measurement methods had been seriously flawed, such generalization would probably 

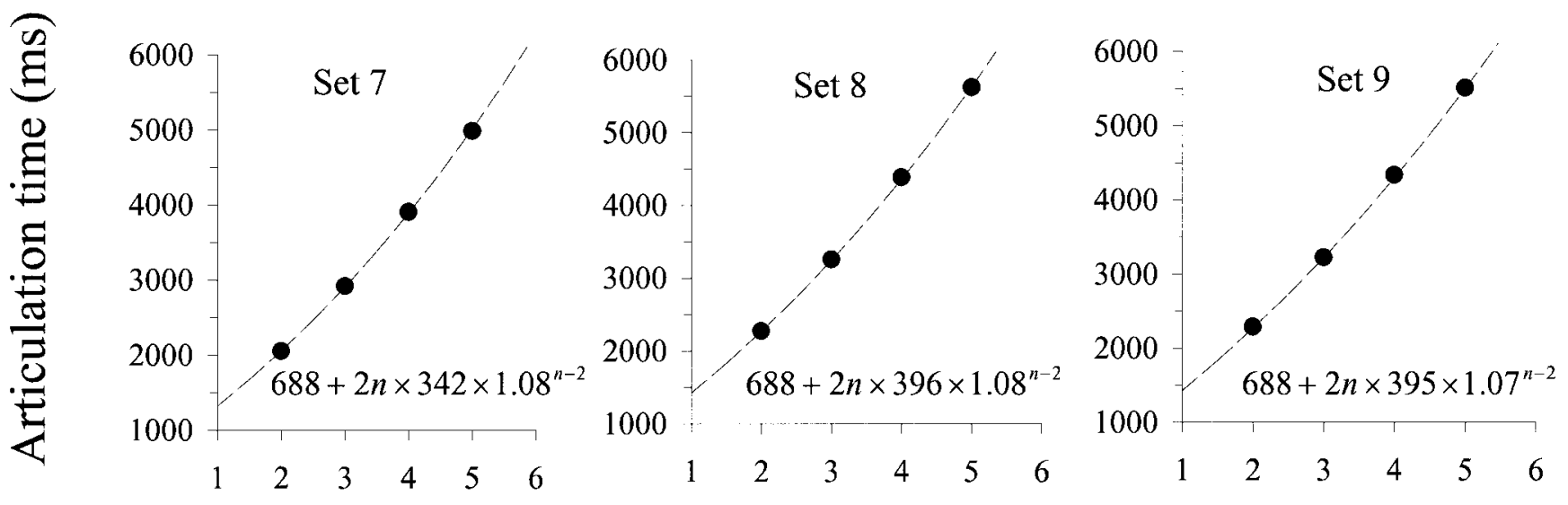

\section{Sequence length}

Figure 4. Observed mean total articulation times (solid circles) and theoretical total articulation-time functions (dashed lines) for each word set and sequence length tested in Experiment 2.

not have been possible here, and the results of Experiment 2 could not have been predicted so accurately from the results of Experiment 1.

\section{General Discussion}

In two experiments on verbal working memory, we have obtained instructive new evidence about the extent to which three theoretically relevant factors affect serial recall accuracy and memory span. Our results show that when articulatory duration and phonological dissimilarity are measured with the methods introduced here, these factors may be extremely accurate predictors of memory spans across word sets. However, contrary to other alternative hypotheses, phonological complexity is a substantially less accurate predictor; it may contribute essentially nothing to serial recall accuracy after articulatory duration and phonological dissimilarity have been taken properly into account. The present evidence has important implications for the status of the phonological-loop model, especially with respect to conclusions based on some previous studies about it.

\section{Reinterpretation of Results From Past VWM Studies}

Although the phonological-loop model provides a good explanation for the results of our two experiments, it is not entirely clear why the results from several past studies on VWM (e.g., Caplan et al., 1992; Caplan \& Waters, 1994; Cowan, Nugent, \& Elliot, 2000; Cowan et al., 1997; Lovatt et al., 2000, 2002; Service, 1998, 2000) appeared to contradict this model. If these studies had measured phonological dissimilarity and articulatory duration through our methods, perhaps many of their results would have supported this model rather than disconfirmed it. To determine whether this may in fact be the case, we next examine each of these studies more carefully.

Caplan et al. (1992). In Experiment 2 of Caplan et al. (1992), performance was compared for two sets of words that were equated on phonological complexity but had either long or short articulatory durations when measured in isolation. Participants' serial recall accuracy was higher for the long words than for the short words. Caplan et al. interpreted these results as evidence against the phonological-loop model, because it predicts that all other things being equal, longer words should yield lower rather than higher serial recall accuracy.

Nevertheless, upon closer inspection, these results appear to be consistent with the phonological-loop model and the findings from our Experiment 1. We found that when participants repetitively articulated Caplan et al.'s (1992) short and long words in memorized variable-length sequences (rather than in isolation), the mean duration of the short words (i.e., Word Set 2) actually exceeded the mean duration of the long words (i.e., Word Set 1). Also, as measured by PSIMETRICA, the phonological dissimilarity of the short words was significantly less than the phonological dissimilarity of the long words. Together, these factors almost perfectly account for the difference in mean memory spans between Caplan et al.'s (Experiment 2) short and long word sets, as the phonological-loop model would predict (see Figure 5). Our findings therefore lead us to conclude that Experiment 2 of Caplan et al. had two limitations: Articulatory duration was measured in a less than ideal way, and the effects of phonological dissimilarity were not taken fully into account.

Still it remains unclear how such limitations could underlie some other results of Caplan et al. (1992). In their Experiment 3, they compared participants' performance for two more sets of words that were equated on phonological complexity but were nominally either difficult or easy to articulate. Confirming this latter difference, the difficult words yielded a longer mean duration than did the easy words when a confederate speaker articulated them in isolation. However, participants' serial recall accuracy was virtually equal $(71.3 \%$ and $71.4 \%$, respectively) for the difficult and easy words, seemingly contrary to the phonological-loop model, which predicts that in this case, serial recall accuracy should have been lower for the difficult words.

These latter results cannot be explained simply by less than ideal measurement of articulatory duration or neglect to take account of countervailing phonological-dissimilarity effects. During our Experiment 1 , when we measured the articulatory durations for the difficult and easy words in memorized variable-length sequences, 


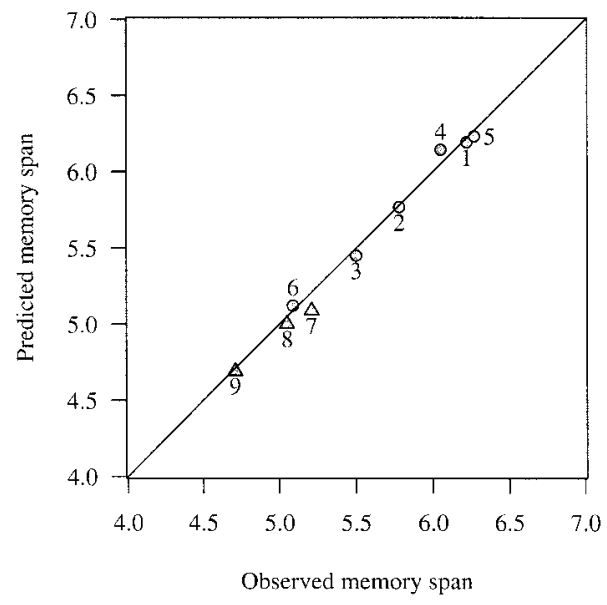

Figure 5. Observed and predicted mean memory spans based on the regression equation. Shaded circles represent the word sets used in Experiment 1; shaded triangles represent the word sets used in Experiment 2. Numerals indicate which word sets yielded the individual pairs of observed and predicted memory spans.

the difficult (Word Set 3) words yielded somewhat longer durations than did the easy (Word Set 4) words, consistent with what Caplan et al. (1992, Experiment 3) found. Also, PSIMETRICA revealed that the difficult words of Word Set 3 were less phonologically dissimilar to each other than were the easy words of Word Set 4. Thus, according to the phonological-loop model, serial recall accuracy should have been lower for the difficult words than for the easy words. Indeed, the results from our Experiment 1 confirmed this prediction. On the other hand, however, the results of Caplan et al. (Experiment 3) differ from ours, because they found that the difficult and easy words yielded essentially equal serial recall accuracy.

Although it may appear at first that this discrepancy is simply a statistical fluke, we believe these results may have a different explanation. Perhaps some aspects of Caplan et al.'s (1992, Experiment 3) procedure discouraged participants from using iterative verbal rehearsal to perform the serial recall task and instead induced participants either to adopt other idiosyncratic (nonverbal) rehearsal strategies or to forego rehearsal entirely. These explanations seem plausible because Caplan et al. (Experiment 3) required serial recall to be made through picture pointing (rather than vocal responses), and their participants were not instructed to rehearse articulatorily. Thus, the procedure used by Caplan et al. (Experiment 3) lacked prerequisite features that may be essential to testing the phonological-loop model fairly, so it is not surprising that they found relatively little difference between serial recall accuracy for their difficult and easy words.

Caplan and Waters (1994). This reinterpretation is supported by subsequent findings that Caplan and Waters (1994) obtained. They repeated Experiment 3 of Caplan et al. (1992) with the same difficult and easy words but measured the accuracy of immediate vocal serial recall for these words, instead of requiring recall to be made through picture-pointing responses. In this modified replication, whose procedure probably encouraged articulatory rehearsal more than before, serial recall accuracy was significantly lower for the difficult words than for the easy words $(64.1 \%$ vs. $72.8 \%$ ). This finding is like what we obtained for these words (see
Table 6), supporting the phonological-loop model and our reinterpretation of the previous atypical results reported by Caplan et al. (Experiment 3).

Cowan et al. (1997). Cowan et al. (1997) obtained results that suggested both phonological complexity and articulatory duration affect serial recall accuracy. In their experiment, participants were presented sequences of either simple (one-syllable) or complex (two-syllable) printed words. During both presentation and later (backward) recall, there were visual timing cues with either short (about $500 \mathrm{~ms}$ ) or long (about $900 \mathrm{~ms}$ ) durations, and the participant was instructed to speak each word of a sequence aloud so that it matched the durations of these signals. Consequently, quasiorthogonal combinations of both articulatory duration and phonological complexity occurred here. Cowan et al. (Experiment 2) found that serial recall accuracy was significantly lower when articulatory durations were long rather than short and that the complex words yielded higher serial recall accuracy than did the simple words.

Without further elaboration, the phonological-loop model cannot explain this beneficial positive effect of phonological complexity on serial recall accuracy. Consequently, Cowan et al. (1997) suggested that this model is either incomplete or incorrect. They proposed that other theoretical constructs, perhaps involving principles of interference rather than time-based decay, are needed to account for serial recall accuracy in their experiments.

However, the apparent positive effect of phonological complexity found by Cowan et al. (1997) may have stemmed from differences in phonological dissimilarity that were confounded with the observed differences in phonological complexity. Evidence for this possibility can be found in Table 10, in which we have used PSIMETRICA to quantify the phonological dissimilarity of both the simple and complex words of Cowan et al. According to this quantification, it appears that the syllable onsets of these complex words were significantly more dissimilar to each other than were those of the simple words ( 0.419 vs. 0.365 , respectively). Furthermore, the complex words had syllable nuclei that were significantly more dissimilar to each other than were those of the simple words $(0.261$ vs. 0.204 , respectively). In contrast, the syllable codas of the complex words were slightly less dissimilar to each other than were the codas of the simple words $(0.252$ vs. 0.272 ,

Table 10

Phonological Dissimilarity of Each Syllable Constituent for the Word Sets Used by Cowan et al. (1997) and Lovatt et al. (2000)

\begin{tabular}{llll}
\hline & \multicolumn{3}{c}{ Phonological dissimilarity } \\
\cline { 2 - 4 } & Onset & Nucleus & Coda \\
\hline Source and word set & & & \\
\hline $\begin{array}{l}\text { Cowan et al. (1997) } \\
\quad \text { Simple }\end{array}$ & 0.365 & 0.204 & 0.272 \\
$\quad$ Complex & 0.419 & 0.261 & 0.252 \\
Lovatt et al. (2000) & & & \\
$\quad$ Experiment 1 & & & \\
$\quad$ Short & 0.318 & 0.108 & 0.200 \\
$\quad$ Long & 0.337 & 0.169 & 0.235 \\
$\quad$ Experiment 2 & & & \\
$\quad$ Short & 0.348 & 0.148 & 0.164 \\
$\quad$ Long & 0.297 & 0.240 & 0.278 \\
$\quad$ Experiment 3 & & & \\
$\quad$ Short & 0.334 & 0.159 & 0.213 \\
$\quad$ Long & 0.397 & 0.193 & 0.178 \\
\hline
\end{tabular}


respectively). Thus, on average, the complex words seem to have been more phonologically dissimilar to each other than were the simple words. Given this systematic variation across these two types of words, our previous regression analysis (the regression equation) implies that phonological-dissimilarity effects may have contributed an advantage of at least 0.3 memory-span units for the complex words over simple words. Such contributions are sufficiently large to suspect that the nominal phonological-complexity effect found by Cowan et al. may have stemmed mostly from a confounding with phonological dissimilarity.

Cowan, Nugent, Elliot, and Geer (2000). Cowan, Nugent, Elliot, and Geer (2000) conducted a subsequent study that provided new insights about this previous experiment. In this study, they used the same stimuli and paced recall procedure as before (Cowan et al., 1997, Experiment 2) but required forward instead of backward serial recall. Under these modified conditions, serial recall accuracies conformed more closely to predictions of the phonological-loop model, which is what should happen if the requirement of forward serial recall especially encourages participants to use incremental cyclic articulatory rehearsal.

Specifically, Cowan, Nugent, Elliot, and Geer (2000) found that with forward serial recall, there were two changes in their results compared with what were reported previously (cf. Cowan et al., 1997, Experiment 2). Longer articulatory durations during the presentation and recall phases of each trial yielded lower recall accuracies at all of the serial positions of the word sequences, as the phonological-loop model would ordinarily predict. Also, unlike before, when participants' articulatory durations during sequence presentation and forward serial recall were approximately equated for simple and complex words, the complex words did not yield higher serial recall accuracies. Thus, Cowan, Nugent, Elliot, and Geer's (2000) results provide additional strong support for the phonological-loop model.

Service (1998). Like other researchers (e.g., Cowan, Nugent, Elliot, \& Geer, 2000; Cowan et al., 1997), Service (1998) tried to separate the effects of articulatory duration and phonological complexity on serial recall accuracy. In her experiments, participants recalled sequences of Finnish pseudowords that were (a) simple disyllables with nominally short articulatory durations, (b) simple disyllables with nominally long articulatory durations, or (c) complex trisyllables with long articulatory durations. Articulatory durations of the simple pseudowords were manipulated by exploiting the fact that Finnish has phonemes whose durations are either short or long but whose other phonological features are supposedly identical. Consequently, the two simple pseudoword sets were identical except for the difference in the lengths of their vowels. However, despite this difference, they produced nearly equal levels of serial recall accuracy, whereas the complex trisyllabic pseudowords yielded much lower recall accuracy. Service therefore concluded that phonological complexity (but not articulatory duration) influences performance in VWM tasks. She also concluded that investigators should abandon the phonological-loop model in favor of some other theoretical account (e.g., one involving interference and redintegrative mechanisms instead of articulatory rehearsal and time-based decay).

Yet this conclusion may be unwarranted, because in Service's (1998) experiments, articulatory durations were not measured under conditions relevant to the phonological-loop model. She measured the articulatory durations of Finnish pseudowords by having participants read visually presented lists and found that the simple long pseudowords yielded much longer (31\%) articulatory durations than did the simple short pseudowords. However, it is likely that this list-reading task imposed certain demand characteristics on the participants, compelling them to speak the two sets of simple pseudowords at distinctively different rates, even if they could rehearse these pseudowords at the same rate during the serial recall task.

To substantiate this possibility, we note that Service (1998) also measured participants' recall durations for the pseudoword sequences in the serial recall task. On the basis of this measurement, which may approximate the ideal method more closely than list reading does, there was only a $7.7 \%$ difference between the durations of the simple short and simple long pseudowords. This difference was small, even though participants were required to extend their overt articulation of the pseudowords with longer vowels to be scored correctly. So quite possibly, when they were not compelled by experimental demands to speak these words at different rates, the participants may have rehearsed the long and short pseudowords at nearly the same rate.

Accordingly, Service's (1998) claims about the importance of phonological-complexity effects on serial recall accuracy require qualification. Because her experiments used unfamiliar pseudowords instead of familiar words for constructing to-bememorized sequences, they may have induced participants to adopt atypical encoding, rehearsal, or recall strategies that magnify the effect of phonological complexity far beyond what occurs for real words under more typical conditions. If so, then the phonological-loop model may still usually provide a veridical account of serial recall accuracy, and further research will be needed to assess phonological complexity's relevance for VWM.

Lovatt et al. (2000). Following Service (1998), these investigators conducted three experiments with the immediate serial recall task to test the phonological-loop model. Each experiment involved two sets of disyllabic words that differed in their nominal mean articulatory durations (short vs. long) but were putatively matched with respect to several other linguistic variables (e.g., frequency, familiarity, phonological dissimilarity, number of phonemes, and semantic associations). Although the word sets changed from one experiment to the next, other aspects of Lovatt et al.'s (2000) designs and procedures were essentially identical across their experiments. In particular, articulatory durations were always measured for isolated words and for words read from lists. ${ }^{19}$ Phonological dissimilarities between the words of each set were measured by having participants rate them on a scale ranging from 1 (not similar) to 5 (very similar), as in some other studies (e.g., Baddeley \& Andrade, 1994; Caplan \& Waters, 1994). These ratings suggested that the mean phonological dissimilarity between the long words almost exactly equaled the mean phonological dissimilarity between the corresponding short words.

Nevertheless, Lovatt et al. (2000) obtained inconsistent articulatory-duration effects on serial recall accuracies. During their first experiment, the long words yielded a reliably higher recall accuracy than did the short words $(65.1 \%$ vs. $60.7 \%$, respec-

\footnotetext{
${ }^{19}$ During Lovatt et al.'s (2000) second and third experiments, articulatory durations were also measured for words in short, constant-length (two-word) repeated sequences. These durations approximately equaled those obtained through list reading, and because of reasons outlined earlier, they may have been less than ideal for testing the phonological-loop model.
} 
tively, with auditory stimuli and vocal responses), whereas the long and short words of their second experiment yielded virtually equal recall accuracies ( $58.5 \%$ vs. $58.7 \%$, respectively). Only during Lovatt et al.'s third experiment did the long words yield a reliably lower recall accuracy than the short words $(65.5 \%$ vs. $70.7 \%$, respectively) as the phonological-loop model would ordinarily predict. Given such apparent empirical inconsistency, Lovatt et al. strongly questioned the general veracity of this model's assumptions.

However, the experiments of Lovatt et al. (2000) have many of the same limitations as the previous experiments that we have discussed. For example, these investigators did not measure the articulatory durations of words in memorized variablelength sequences. Consequently, it is unclear whether the articulatory durations of these words differed in ways that are relevant to the phonological-loop model. In addition, phonological dissimilarity was assessed by Lovatt et al. through a subjective rating procedure. Because subjective ratings may not be sensitive enough to detect relevant differences in phonological dissimilarity that can affect serial recall accuracy, perhaps the word sets of Lovatt et al. were not actually equated with respect to phonological dissimilarity.

To examine this possibility further, we measured the phonological dissimilarity of Lovatt et al.'s (2000) word sets (see Table 10) using PSIMETRICA. For the short words and long words of their first experiment, the dissimilarities between the respective onsets, nuclei, and codas of the long words were uniformly greater than the dissimilarities between those of the short words. From the perspective of the phonological-loop model and our previous regression analysis (the regression equation), this greater dissimilarity between the long words could have counteracted the effect of articulatory duration and produced a higher serial recall accuracy than did the short words.

In Lovatt et al.'s (2000) second experiment, the phonological dissimilarities between the nuclei and codas of the long words were again greater than the corresponding dissimilarities for the short words. However, these long words had onsets whose phonological dissimilarity was considerably less than that of the short words (see Table 10). Such opposing degrees of phonological dissimilarity might explain why the long and short words of Lovatt et al.'s second experiment yielded almost equal serial recall accuracies.

In their third experiment, the long words yielded lower serial recall accuracy than did the short words. This happened even though the long words again tended to be more dissimilar than were the short words (see Table 10). So in this last case, the difference between their mean articulatory durations was apparently great enough to counteract the effect of phonological dissimilarity.

Lovatt et al. (2002). Finally, across four experiments, Lovatt et al. (2002) reported two more cases (Experiments 1 and 3) in which sequences of long words were recalled as well as sequences of short words and one case in which sequences of long words were recalled more accurately than sequences of short words (Experiment 4). Experiments 1 and 3 used a subset of the stimuli from Experiment 2 of Lovatt et al. (2000), so they are open to many of the same caveats mentioned before. Experiment 4 involved the short and long words of Caplan et al. (1992), and the results were similar to what was found by Caplan et al., Caplan and Waters (1994), and us (Word Sets 1 and 2 of Experiment 1).
Consequently, these experiments by Lovatt et al. (2002) provided no further compelling evidence against the phonological-loop model.

Summary of reinterpreted experiments. When initially examined, previous experiments (i.e., Caplan et al., 1992; Caplan \& Waters, 1994; Cowan, Nugent, Elliot, \& Geer, 2000; Cowan et al. 1997; Lovatt et al., 2000, 2002; Service, 1998, 2000) each appeared to offer evidence against the phonological-loop model. However, taken as a whole, they do not support any clear alternative theory. Furthermore, when we examined their results through the lenses of PSIMETRICA and our articulatory-duration measurement method, it appears that the phonological-loop model is consistent with all of them. This model can even explain why some of the attempts to replicate these past results have failed.

\section{Status of the Phonological-Loop Model}

On the basis of our results from Experiments 1 and 2, as well as our reinterpretation of previous experiments, several core assumptions of the phonological-loop model have received additional strong empirical support.

Phonological coding of stored word sequences. The phonological-loop model assumes that during VWM tasks such as immediate serial recall, the words of memorized sequences are coded and stored as temporary phonological representations (Baddeley, 1986). In support of this assumption, we have found that precise quantitative measurement of phonological dissimilarity through PSIMETRICA can help predict serial recall accuracy. Such predictability is what would be expected if the temporary stored representations of words are closely tied to information used by the vocal articulators for producing covert and overt speech during rehearsal, as in the phonological-loop model.

Information loss through time-based decay. A second related assumption of this model is that the loss of information from VWM occurs through time-based decay. Because of such decay, the principal limit on the functional capacity of VWM may be the time required to refresh the memory traces of stored items. Although decay may not be the only factor that limits VWM storage capacity, there is little compelling evidence that phonological complexity plays a significant role here.

Memory-trace retention by strategic articulatory rehearsal. The phonological-loop model also assumes that the retention of memory traces entails strategic articulatory rehearsal. This assumption, like the preceding one, is supported by our finding that articulatory durations measured for words in memorized sequences are reliable predictors of memory spans, whereas articulatory durations measured for isolated words are not. On the other hand, if articulatory rehearsal were irrelevant to memory-trace retention in VWM, then how we measured the articulatory durations of words should have been irrelevant, and memory spans should have been uncorrelated with all of these duration measures. The absence of duration effects on serial recall accuracy when recall involves picture pointing rather than vocal responses (Caplan et al., 1992, Experiment 3) likewise demonstrates the optional strategic nature of articulatory rehearsal.

\section{New Insights About Articulatory Rehearsal and Phonological-Dissimilarity Effects}

Although the results of Experiments 1 and 2 support several core assumptions of the phonological-loop model, they also pro- 
vide additional insights about ways in which this model should be refined and elaborated further. For example, we found that under the present conditions, memory spans could be predicted reliably by the phonological dissimilarity between syllable onsets but that the dissimilarity between syllable rhymes was an unreliable predictor. This may have occurred because participants' articulation during rehearsal truncated some syllables to cycle through the memorized word sequences more rapidly. Such truncation may have nullified the phonological features in the syllable rhymes, making the different features of the onsets relatively more important for the effect of phonological dissimilarity on serial recall accuracy. If so, then the possible contributions of these variations in rehearsal strategies must be factored into future formulations of the phonological-loop model.

A second insight from our Experiment 1 about articulatory rehearsal is that the measured durations of words depend on how participants have to articulate them. On average, words articulated in memorized sequences have much shorter durations than do words articulated in isolation. Consequently, for certain pairs of word sets, the difference between the mean articulatory durations of their words may be reliably positive or negative, depending on whether sequential or isolated articulation is involved. This dependence can occur because articulatory rehearsal entails relatively rapid utterances in which syllable rhymes are presumably truncated more than syllable onsets, thereby changing which parts of the syllables contribute most to the measured durations of words. ${ }^{20}$

A third insight, obtained through our reinterpretation of previous studies, is that procedures for testing VWM can influence what strategies participants use for performing the memory tasks. For example, some testing procedures may discourage the use of articulatory rehearsal (e.g., those in Experiment 3 of Caplan et al., 1992, and in Cowan et al., 1997). When this happens, it will yield a diminished effect of articulatory duration on recall accuracy compared with experiments that encourage participants' use of such rehearsal (e.g., as in our experiments, as well as in Caplan \& Waters, 1994, and in Cowan, Nugent, \& Elliot, 2000).

Taken together, our new insights indicate that the effects of articulatory duration and phonological dissimilarity, often viewed as bellwethers of the phonological-loop model, will not necessarily occur whenever word sets differ in terms of these factors. For these factors to affect performance in a memory task, the task must encourage the participant to use a phonological code, and the participant must engage in articulatory rehearsal. In addition, for the effects of these factors to be detected, suitable measurement methods must be used. If these requirements are not met, then the phonological-loop model does not predict that articulatory duration or phonological dissimilarity will have reliable effects. The full range of conditions under which this model's predictions are applicable remains to be determined.

\footnotetext{
${ }^{20}$ A similar conclusion was reached by Dell and Repka (1992). They had participants report speech errors made during either a covert or an overt articulation task. For both tasks, the majority of reported speech errors occurred in initial consonants. Fewer errors were reported for noninitial consonants and vowels, and they occurred mainly during the overt speech condition. These results led Dell and Repka to conclude that the phonological representation used in covert speech decreased in strength toward the end of each word. This conclusion differs slightly from ours because we propose that the rhyme of each syllable is truncated (rather than
}

the entire latter part of each word). However, like us, Dell and Repka concluded that such truncation is probably a strategic response to task conditions, and so its nature might depend on specific demands of the task.

\section{References}

Anderson, J. R., \& Matessa, M. (1997). A production system theory of serial memory. Psychological Review, 104, 728-748.

Baddeley, A. D. (1966). Short-term memory for word sequences as a function of acoustic, semantic, and formal similarity? Quarterly Journal of Experimental Psychology, 18, 362-365.

Baddeley, A. D. (1968). How does acoustic similarity influence short-term memory? Quarterly Journal of Experimental Psychology, 20, 249-264.

Baddeley, A. D. (1986). Working memory. Oxford, England: Oxford University Press.

Baddeley, A. D. (1992, January 8). Working memory. Science, 255, 566-569.

Baddeley, A. D., \& Andrade, J. (1994). Reversing the word-length effect: A comment on Caplan, Rochon, and Waters. Quarterly Journal of Experimental Psychology: Human Experimental Psychology, 47(A), 1047-1054.

Baddeley, A. D., \& Hitch, G. J. (1974). Working memory. In G. H. Bower (Ed.), Recent advances in learning and motivation (Vol. 8, pp. 47-90). New York: Academic Press.

Baddeley, A. D., Lewis, V., \& Vallar, G. (1984). Exploring the articulatory loop. Quarterly Journal of Experimental Psychology: Human Experimental Psychology, 36(A), 233-252.

Baddeley, A. D., \& Logie, R. H. (1999). Working memory: The multiplecomponent model. In A. Miyake \& P. Shah (Eds.), Models of working memory: Mechanisms of active maintenance and executive control (pp. 28-61). New York: Cambridge University Press.

Baddeley, A. D., Thomson, N., \& Buchanan, M. (1975). Word length and the structure of short-term memory. Journal of Verbal Learning and Verbal Behavior, 14, 575-589.

Baddeley, A. D., \& Wilson, B. (1985). Phonological coding and short-term memory in patients without speech. Journal of Memory and Language, 14, 575-589.

Basso, A., Spinnler, H., Vallar, G., \& Zanobio, E. (1982). Left hemisphere damage and selective impairment of auditory verbal short-term memory: A case study. Neuropsychologia, 20, 263-274.

Brown, G. D., \& Hulme, C. (1995). Modeling item length effects in memory span: No rehearsal needed? Journal of Memory and Language, $34,594-624$.

Cantor, J., \& Engle, R. W. (1993). Working-memory capacity as long-term memory activation: An individual-differences approach. Journal of Experimental Psychology: Learning, Memory, and Cognition, 19, 11011114.

Caplan, D., Rochon, E., \& Waters, G. S. (1992). Articulatory and phonological determinants of word length effects in span tasks. Quarterly Journal of Experimental Psychology: Human Experimental Psychology, 45(A), 177-192.

Caplan, D., \& Waters, G. S. (1994). Articulatory length and phonological similarity in span tasks: A reply to Baddeley and Andrade. Quarterly Journal of Experimental Psychology: Human Experimental Psychology, 47(A), 1055-1062.

Chomsky, N., \& Halle, M. (1968). The sound pattern of English. New York: Harper \& Row.

Clark, H. H., \& Clark, E. V. (1977). Psychology and language. New York: Harcourt Brace Jovanavich.

Colle, H. A., \& Welsh, A. (1976). Acoustic masking in primary memory. Journal of Verbal Learning and Verbal Behavior, 15, 17-32.

Coltheart, M. (1981). The MRC psycholingistic database. Quarterly Journal of Experimental Psychology: Human Experimental Psychology, 33(A), 497-505.

Conrad, R., \& Hull, A. J. (1964). Information, acoustic confusion, and memory span. British Journal of Psychology, 55, 429-432. 
Cowan, N. (1992). Verbal memory span and the timing of spoken recall. Journal of Memory and Language, 31, 668-684.

Cowan, N. (1999). An embedded-processes model of working memory. In A. Miyake \& P. Shah (Eds.), Models of working memory: Mechanisms of active maintenance and executive control (pp. 62-101). New York: Cambridge University Press

Cowan, N., Nugent, L. D., \& Elliot, E. M. (2000). Memory-search and rehearsal processes and the word length effect in immediate serial recall: A synthesis in reply to Service. Quarterly Journal of Experimental Psychology: Human Experimental Psychology, 53(A), 666670.

Cowan, N., Nugent, L. D., Elliot, E. M., \& Geer, T. (2000). Is there a temporal basis of the word length effect? A response to Service (1998). Quarterly Journal of Experimental Psychology: Human Experimental Psychology, 53(A), 647-660.

Cowan, N., Wood, N. L., Nugent, L. D., \& Treisman, M. (1997). There are two word-length effects in verbal short-term memory: Opposed effects of duration and complexity. Psychological Science, 8, 290-295.

Cowan, N., Wood, N. L., Wood, P. K., Keller, T. A., Nugent, L. D., \& Keller, C. V. (1998). Two separate verbal processing rates contributing to short-term memory span. Journal of Experimental Psychology: General, 127, 141-160.

Dell, G. S., \& Repka, R. J. (1992). Errors in inner speech. In B. J. Baars (Ed.), Experimental slips and human error: Exploring the architecture of volition (pp. 237-262). New York: Plenum Press.

Della Sala, S., Logie, R. H., Marchetti, C., \& Wynn, V. (1991). Case studies in working memory: A case for single cases? Cortex, 27, 169191.

D'Esposito, M., \& Postle, B. (2000). Neural correlates of component processes of working memory: Evidence from neuropsychological and pharmacological studies. In S. Monsell \& J. Driver (Eds.), Attention \& performance XVIII: Control of cognitive processes (pp. 579-602). Cambridge, MA: MIT Press

Dosher, B. A., \& Ma, J. (1998). Output loss or rehearsal loop? Output-time versus pronunciation-time limits in immediate recall for forgetting matched materials. Journal of Experimental Psychology: Learning, Memory, and Cognition, 24, 316-335.

Drewnowski, A. (1980). Attributes and priorities in short-term recall: A new model of memory span. Journal of Experimental Psychology: General, 109, 208-250.

Estes, W. K. (1972). An associative basis for coding and organization in memory. In A. W. Melton \& E. Martin (Eds.), Coding processes in human memory (pp. 161-190). Washington, DC: Winston.

Frisch, S. A. (1997). Against underspecification in speech errors. Studies in the Linguistic Sciences, 27, 79-97.

Fudge, E. C. (1969). Syllables. Journal of Linguistics, 5, 253-286.

Gupta, P., \& MacWhinney, B. (1995). Is the articulatory loop articulatory or auditory? Reexamining the effects of concurrent articulation on immediate serial recall. Journal of Memory and Language, 34, 63-88.

Hartley, T., \& Houghton, G. (1996). A linguistically constrained model of short-term memory for non-words. Journal of Memory and Language, $35,1-31$.

Hodgeman, C. D. (1941). Mathematical tables from handbook of chemistry and physics (7th ed.). Cleveland, $\mathrm{OH}$ : Chemical Rubber Publishing Company.

Hulme, C., Roodenrys, S., Schweickert, R., Brown, G. D. A., Martin, S., \& Stuart, G. (1997). Word-frequency effects on short-term memory tasks: Evidence for a redintegration process in immediate serial recall. Journal of Experimental Psychology: Learning, Memory, and Cognition, 23, $1217-1232$

Kieras, D. E., Meyer, D. E., Mueller, S., \& Seymour, T. (1999). Insights into working memory from the perspective of the EPIC architecture for modeling skilled perceptual-motor and cognitive human performance. In A. Miyake \& P. Shah (Eds.), Models of working memory: Mecha- nisms of active maintenance and executive control (pp. 183-223). New York: Cambridge University Press.

Kučera, H., \& Francis, W. N. (1967). Computational analysis of presentday American English. Providence, RI: Brown University Press.

Landauer, T. K. (1962). The rate of implicit speech. Perceptual and Motor Skills, 15, 646.

Laughery, K. R., \& Pinkus, A. L. (1970). A simulation model of short-term memory: Parameter sensitivity studies and implications for two current issues. Journal of Mathematical Psychology, 7, 554-571.

Levy, B. A. (1971). Role of articulation in auditory and visual short-term memory. Journal of Verbal Learning and Verbal Behavior, 10, 123-132.

Lewandowsky, S., \& Murdock, B. B. (1989). Memory for serial order. Psychological Review, 96, 25-57.

Logie, R. H., Della Sala, S., Laiacona, M., \& Chalmers, P. (1996). Group aggregates and individual reliability: The case of verbal short-term memory. Memory \& Cognition, 24, 305-321.

Longoni, A. M., Richardson, A. T. E., \& Aiello, A. (1993). Articulatory rehearsal and phonological storage in working memory. Memory \& Cognition, 21, 11-22.

Lovatt, P., Avons, S. E., \& Masterson, J. (2000). The word-length effect and disyllabic words. Quarterly Journal of Experimental Psychology: Human Experimental Psychology, 53(A), 1-22.

Lovatt, P., Avons, S. E., \& Masterson, J. (2002). Output decay in immediate serial recall: Speech time revisited. Journal of Memory and Language, 46, 227-243.

Mazzoni, D. (2001). Audacity (Version 0.97) [Computer software]. Available from http://audacity.sf.net

McCullagh, P., \& Nelder, J. A. (1989). Generalized linear models (2nd ed.). London: Chapman \& Hall.

Miller, G. A. (1956). The magical number seven, plus or minus two: Some limits to our capacity for processing information. Psychological Review, 63, 81-97.

Miyake, A., \& Shah, P. (Eds.). (1999). Models of working memory: Mechanisms of active maintenance and executive control. New York: Cambridge University Press.

Murdock, B. B. (1974). Human memory: Theory and data. Potomac, MD: Erlbaum.

Murdock, B. B. (1993). TODAM2: A model for the storage and retrieval of item, associative, and serial-order information. Psychological Review, 100, 183-203.

Murray, D. J. (1967). The role of speech responses in short-term memory. Canadian Journal of Psychology, 21, 263-276.

Murray, D. J. (1968). Articulation and acoustic confusability in short-term memory. Journal of Experimental Psychology, 78, 679-684.

Nairne, J. S. (1990). A feature model of immediate memory. Memory \& Cognition, 18, 251-269.

O'Grady, W., \& Dobrovrolsky, M. (1992). Contemporary linguistic analysis (2nd ed.). Toronto, Ontario, Canada: Copp Clark Pitman.

Page, M. P. A., \& Norris, D. (1998). The primary model: A new model of immediate serial recall. Psychological Review, 105, 761-768.

Posner, M. I., \& Konick, A. F. (1966). On the role of interference in short-term memory. Journal of Experimental Psychology, 72, 221231.

Rosenbaum, D. A., Gordon, A. M., Stillings, N. A., \& Feinstein, M. H. (1987). Stimulus-response compatibility in the programming of speech. Memory \& Cognition, 15, 217-224.

Salamé, P., \& Baddeley, A. D. (1982). Disruption of short-term memory by unattended speech: Implications for the structure of working memory. Journal of Verbal Learning and Verbal Behavior, 21, 150-164.

Schweickert, R., \& Boruff, B. (1986). Short-term memory capacity: Magic number or magic spell? Journal of Experimental Psychology: Learning, Memory, and Cognition, 12, 419-425.

Schweickert, R., Guentert, L., \& Hersberger, L. (1990). Phonological similarity, pronunciation rate, and memory span. Psychological Science, $1,74-77$. 
Service, E. (1998). The effect of word length on immediate serial recall depends on phonological complexity, not articulatory duration. Quarterly Journal of Experimental Psychology: Human Experimental Psychology, 51(A), 283-304.

Service, E. (2000). Phonological complexity and word duration in immediate recall: Different paradigms answer different questions. A comment on Cowan, Nugent, Elliot, and Geer. Quarterly Journal of Experimental Psychology: Human Experimental Psychology, 53(A), 661-665.

Shallice, T., \& Warrington, E. K. (1970). Independent functioning of verbal memory stores: A neuropsychological study. Quarterly Journal of Experimental Psychology, 22, 261-273.

Sperling, G. (1967). Successive approximations to a model for short-term memory. Acta Psychologica, 27, 285-292.

Standing, L., Bond, B., Smith, P., \& Isely, C. (1980). Is the immediate memory span determined by subvocalization rate? British Journal of Psychology, 71, 525-539.

Standing, L., \& Curtis, L. (1989). Subvocalization rate versus other predictors of the memory span. Psychological Reports, 65, 487-495.

Sternberg, S., Monsell, S., Knoll, R. L., \& Wright, C. E. (1978). The latency and duration of rapid movement sequences: Comparisons of speech and typewriting. In G. E. Stelmach (Ed.), Information processing in motor control (pp. 117-152). New York: Academic Press.

Treiman, R., \& Zukowski, A. (1990). Toward an understanding of English syllabification. Journal of Memory and Language, 29, 66-85.

Vitz, P. C., \& Winkler, B. S. (1973). Predicting the judged "similarity of sound" of English words. Journal of Verbal Learning and Verbal Behavior, 12, 373-388.

Waugh, N. C., \& Norman, D. A. (1965). Primary memory. Psychological Review, 72, 89-104.

Wickelgren, W. A. (1965). Distinctive features and errors in short-term memory for English vowels. Journal of the Acoustical Society of America, 38, 583-588.

Wickelgren, W. A. (1966). Distinctive features and errors in short-term memory for English consonants. Journal of the Acoustical Society of America, 39, 388-398.

Wilson, M. (1987). The MRC psycholinguistic database machine usable dictionary (Version 2.0) [Computer software and data file]. Available from http://www.psy.uwa.edu.au/mrcdatabase/uwa_mrc.htm

\section{Appendix A}

\section{Representation of Phonemes}

To measure phonological dissimilarity for a pair of words with PSIMETRICA, we represent each word in terms of its phonemes. Each phoneme is decomposed into values that it has for the following features: (a) vocalic, (b) consonantal, (c) high, (d) back, (e) low, (f) anterior, (g) coronal, (h) round, (i) tense, (j) voice, (k) continuant, (l) nasal, and (m) strident. Vowels do not have values for the voice, continuant, nasal, and strident features; some consonants do not have values for the round and tense features. Table A1 shows the phonological-feature values for a standard set of phonemes as described by Clark and Clark (1977, p. 187) and Chomsky and Halle (1968).

Table A1

Phonological-Feature Values for a Standard Set of Phonemes

\begin{tabular}{|c|c|c|c|c|c|c|c|c|c|c|c|c|c|c|c|c|c|c|c|c|c|c|c|c|c|c|c|}
\hline \multirow[b]{2}{*}{ Phoneme } & \multicolumn{13}{|c|}{ Phonological-feature values } & \multirow[b]{2}{*}{ Phoneme } & \multicolumn{13}{|c|}{ Phonological-feature values } \\
\hline & $\mathrm{a}$ & $\mathrm{b}$ & $\mathrm{c}$ & $\mathrm{d}$ & $\mathrm{e}$ & $\mathrm{f}$ & $\mathrm{g}$ & $\mathrm{h}$ & $\mathrm{i}$ & $\mathrm{j}$ & $\mathrm{k}$ & 1 & $\mathrm{~m}$ & & $\mathrm{a}$ & $\mathrm{b}$ & $\mathrm{c}$ & d & $\mathrm{e}$ & $\mathrm{f}$ & $\mathrm{g}$ & $\mathrm{h}$ & $\mathrm{i}$ & $\mathrm{j}$ & $\mathrm{k}$ & 1 & $\mathrm{~m}$ \\
\hline /i/ & + & - & + & - & - & - & - & - & + & $\mathrm{X}$ & $\mathrm{X}$ & $\mathrm{x}$ & $\mathrm{X}$ & $/ \mathrm{b} /$ & - & + & - & - & - & + & - & $\mathrm{X}$ & $\mathrm{X}$ & + & - & - & - \\
\hline /I/ & + & - & + & - & - & - & - & - & - & $\mathrm{X}$ & $\mathrm{X}$ & $\mathrm{X}$ & $\mathrm{X}$ & $/ \mathrm{t} /$ & - & + & - & - & - & + & + & $\mathrm{X}$ & $\mathrm{X}$ & - & - & - & - \\
\hline /e/ & + & - & - & - & - & - & - & - & + & $\mathrm{X}$ & $\mathrm{x}$ & $\mathrm{x}$ & $\mathrm{X}$ & $/ \mathrm{d} /$ & - & + & - & - & - & + & + & $\mathrm{X}$ & $\mathrm{X}$ & + & - & - & - \\
\hline$|\varepsilon|$ & + & - & - & - & - & - & - & - & - & $\mathrm{X}$ & $\mathrm{X}$ & $\mathrm{X}$ & $\mathrm{X}$ & $/ \check{c} /$ & - & + & + & - & - & - & + & $\mathrm{X}$ & $\mathrm{X}$ & - & - & - & + \\
\hline /æ/ & + & - & - & - & + & - & - & - & + & $\mathrm{X}$ & $\mathrm{X}$ & $\mathrm{x}$ & $\mathrm{X}$ & /j/ & - & + & + & - & - & - & + & $\mathrm{X}$ & $\mathrm{X}$ & + & - & - & + \\
\hline /i/ & + & - & + & + & - & - & - & - & - & $\mathrm{X}$ & $\mathrm{x}$ & $\mathrm{x}$ & $\mathrm{X}$ & $/ \mathrm{k} /$ & - & + & + & + & - & - & - & - & $\mathrm{X}$ & - & - & - & - \\
\hline /ə/ & + & - & - & + & - & - & - & - & - & $\mathrm{X}$ & $\mathrm{x}$ & $x$ & $\mathrm{X}$ & $/ \mathrm{g} /$ & - & + & + & + & - & - & - & - & $\mathrm{X}$ & + & - & - & - \\
\hline $\mid \Lambda /$ & + & - & - & + & + & - & - & - & - & $\mathrm{X}$ & $\mathrm{X}$ & $\mathrm{x}$ & $\mathrm{X}$ & /f/ & - & + & - & - & - & + & - & $\mathrm{X}$ & $\mathrm{X}$ & - & + & - & + \\
\hline /a/ & + & - & - & + & + & - & - & - & + & $\mathrm{X}$ & $\mathrm{x}$ & $\mathrm{x}$ & $\mathrm{X}$ & $/ \mathrm{v} /$ & - & + & - & - & - & + & - & $\mathrm{X}$ & $\mathrm{X}$ & + & + & - & + \\
\hline$/ \mathrm{u} /$ & + & - & + & + & - & - & - & + & + & $\mathrm{X}$ & $\mathrm{x}$ & $\mathrm{X}$ & $\mathrm{X}$ & $/ \theta /$ & - & + & - & - & - & + & + & $\mathrm{X}$ & $\mathrm{X}$ & - & + & - & - \\
\hline /u/ & + & - & + & + & - & - & - & + & - & $\mathrm{X}$ & $x$ & $\mathrm{X}$ & $x$ & /ð/ & - & + & - & - & - & + & + & $\mathrm{X}$ & $\mathrm{X}$ & + & + & - & - \\
\hline$/ \mathrm{o} /$ & + & - & - & + & - & - & - & + & + & $\mathrm{X}$ & $\mathrm{X}$ & $x$ & $\mathrm{X}$ & /s/ & - & + & - & - & - & + & + & $\mathrm{X}$ & $\mathrm{X}$ & - & + & - & + \\
\hline $\mid \mathbf{0} /$ & + & - & - & + & + & - & - & + & - & $\mathrm{X}$ & $\mathrm{X}$ & $\mathrm{X}$ & $\mathrm{X}$ & $|z|$ & - & + & - & - & - & + & + & $\mathrm{X}$ & $\mathrm{X}$ & + & + & - & + \\
\hline /y/ & - & - & + & - & - & - & - & - & - & $\mathrm{X}$ & $\mathrm{X}$ & $\mathrm{x}$ & $\mathrm{x}$ & $/ \int /$ & - & + & + & - & - & - & + & $\mathrm{X}$ & $\mathrm{X}$ & - & + & - & + \\
\hline$/ \mathrm{w} /$ & - & - & + & + & - & - & - & + & - & $\mathrm{X}$ & $x$ & $\mathrm{x}$ & $\mathrm{x}$ & $|3|$ & - & + & + & - & - & - & + & $x$ & $\mathrm{X}$ & + & + & - & + \\
\hline$/ \mathrm{r} /$ & + & + & - & - & - & - & + & $\mathrm{X}$ & $\mathrm{X}$ & + & + & - & - & $/ \mathrm{m} /$ & - & + & - & - & - & + & - & $\mathrm{X}$ & $\mathrm{X}$ & + & - & + & - \\
\hline /1/ & + & + & - & - & - & + & + & $\mathrm{X}$ & $\mathrm{X}$ & + & + & - & - & $/ \mathrm{n} /$ & - & + & - & - & - & + & + & $\mathrm{X}$ & $\mathrm{X}$ & + & - & + & - \\
\hline$/ \mathrm{h} /$ & - & - & - & - & + & - & - & $\mathrm{X}$ & $\mathrm{X}$ & - & + & - & - & $/ \mathrm{y} /$ & - & + & + & + & - & - & - & $x$ & $\mathrm{x}$ & + & - & + & - \\
\hline$/ \mathrm{p} /$ & - & + & - & - & - & + & - & $\mathrm{X}$ & $\mathrm{X}$ & - & - & - & - & & & & & & & & & & & & & & \\
\hline
\end{tabular}

Note. + and - indicate that a feature takes a positive or a negative value, respectively, for a phoneme. $\mathrm{x}$ indicates that a feature has no value for that phoneme. 


\section{Appendix B}

\section{Analysis for Estimation of Mean Articulatory Durations}

The mean articulatory duration of word sequences tends to be a concaveupward function of sequence length (cf. Sternberg et al., 1978). It may also contain contributions of speech preparation and recording biases that are independent of word set and sequence length. Consequently, raw sequencearticulation times should be analyzed with a mathematical model that takes these potential contributions into account

In the present experiments, sequences of between two and five words were repeated twice from memory for multiple word sets (indexed by $k$ ) and multiple participants (indexed by $j$ ). From the results of this procedure, we approximate the total articulation time on each trial by

$$
T=I_{j}+2 \times n \times d_{j k} \times a_{j k}^{n-2} .
$$

Here $I$ is an intercept parameter, $n$ is the sequence length, $d$ is a base articulatory duration per word, and $a$ is a duration-amplification factor. For each combination of word set and participant, our analysis yields an estimate of the mean sequence duration based on $I, d, a$, and $n$.

In this analysis, we assumed that $I$ depends on neither $n$ nor the word set from which a sequence has been constructed. However, $I$ may vary across participants and includes temporal overhead due to sequence repetition as well as any biases contributed by the apparatus or experimenter to the measurement procedure. The base articulatory duration per word, $d$, depends on the words from which a sequence has been constructed. Also, on the right side of Equation B1, the product $2 \times n \times d_{j k} \times a_{j k}^{\mathrm{n}-2}$ embodies contributions due to the $n$-word sequence being repeated twice and the duration per word increasing with $n$. As part of this product, the amplification factor $a_{j k}$ is raised to the $n-2$ power because two-word sequences are the shortest ones for which Equation B1 may hold. ${ }^{\mathrm{B} 1}$

Given the observed total articulation times, the accuracy of Equation B1 can be assessed by calculating a deviation score, $\delta_{i j k}$, for each articulation trial $i$ performed by participant $j$ with a sequence of words constructed from word set $k$. In the calculation,

$$
\delta_{i j k}=\frac{\left(t_{i j k, \text { observed }}-t_{i j k, \text { predicted }}\right)}{n_{i j k}},
$$

where $n_{i j k}$ is the sequence length, and $t_{i j k \text {,predicted }}$ is based on Equation B1. Values of $t_{i j k \text {,predicted }}$ can be derived by minimizing $\Sigma_{i, j, k}\left(\delta_{i j k}\right)^{2}$, which yields a single intercept parameter $I_{j}$ for each participant $j$, as well as an articulatory duration parameter $d_{j k}$ and an amplification parameter $a_{j k}$ for each word set $k$ encountered by participant $j$. At this point, outliers among the values of $t_{i j k, \text { observed }}$ can be removed by excluding trials whose values of $\delta_{i j k}$ differ by more than some number (e.g., 2.5) of standard deviations from participant $j$ 's mean $\delta_{i j k}$.

On the basis of the parameters $a_{j k}$ and $d_{j k}$, the mean articulatory duration per word in memorized sequences can be estimated for each word set. This involves two further steps. First, for each word set $k$ with which participant $j$ performs, the mean articulatory duration per word, $D_{j k}$, is estimated as

$$
D_{j k}=\sum_{n=2}^{5}\left(d_{j k} \times a_{j k}^{n-2}\right) / 4,
$$

where $d_{j k}$ is the base word duration, and $a_{j k}$ is the duration-amplification parameter in Equation B1 when participant $j$ and word set $k$ are involved.

Second, for each word set $k$, the values of $D_{j k}$ from Equation B3 are averaged across participants, yielding $D_{k}$, the mean articulatory duration per word in sequences constructed with this word set. These final $D_{k}$ values are then taken into account as part of assessing articulatory-duration effects on the memory-span data.

Equation B3 is used to estimate $D_{j k}$ because during presentation of to-be-recalled words in the serial recall task, rehearsal of short subsequences presumably precedes rehearsal of longer subsequences (Kieras et al., 1999). Also, by using Equation B3, we take into account that the articulatory duration per word increases with the length of the subsequences being rehearsed successively. Equation B3 omits the intercept of Equation B1 because $I$ is presumably independent of the individual word durations and only embodies contributions from ancillary sources that do not vary systematically as a function of sequence length.

For purposes of interpreting the results from this subsequent assessment, it should be stressed that using Equation B1 to help measure the mean articulatory durations of words in memorized sequences has some especially significant benefits. Doing so enables us to remove apparatus and experimenter biases that may have contributed to the observed total articulation times but that should have been excluded from estimates of the mean articulatory durations for words in memorized sequences. Consequently, regardless of whether these durations come from sources such as manual stopwatch timing or examination of acoustic waveform records, they may be reasonably veridical insofar as they conform to Equation B1 and are quantified appropriately in terms of its parameters (e.g., see Footnote 18).

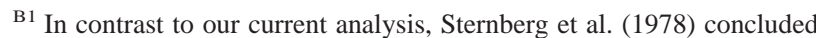
that total articulation times for memorized word sequences could be fit well by a quadratic function of sequence length. However, we have found that an exponential function (i.e., Equation B1) provides more interpretable parameter values under the conditions of our experiments, and it fits our data slightly better than does a quadratic function. Yet under other circumstances, given that $1+n \times a \approx(1+a)^{n}$ when $a$ is small (Hodgeman, 1941), it would be difficult to distinguish Equation B1 empirically from a quadratic function. 


\section{Appendix C}

Word Sets Used in Experiments 1 and 2

\begin{tabular}{|c|c|c|c|c|c|}
\hline \multicolumn{2}{|c|}{ Set 1 (long) } & \multicolumn{2}{|c|}{ Set 2 (short) } & \multicolumn{2}{|c|}{ Set 3 (difficult) } \\
\hline Word & Phonemic transcription & Word & Phonemic transcription & Word & Phonemic transcription \\
\hline crayon & /'kre yən/ & carrot & /'ke rət/ & crow & /'kro/ \\
\hline vacuum & /'væ kium/ & bullet & /'bu lət/ & stew & /'stu/ \\
\hline sirloin & /'sər loin/ & ladder & /'læ dər/ & glue & /'glu/ \\
\hline spider & /'spai dər/ & devil & /'d $\varepsilon$ vəl// & tree & /'tri/ \\
\hline balloon & /bə 'lun/ & picnic & /'pık nIk/ & tray & /'tre/ \\
\hline baby & /'bei bi/ & ticket & /'ti kit/ & draw & /'dra/ \\
\hline tower & /'tau wər/ & zipper & /'zI pər/ & snow & /'sno/ \\
\hline orange & /'ou rənj/ & cabin & /'kæ bən/ & & \\
\hline \multicolumn{2}{|c|}{ Set 4 (easy) } & \multicolumn{2}{|c|}{ Set 5 (short) } & \multicolumn{2}{|c|}{ Set 6 (long) } \\
\hline Word & Phonemic transcription & Word & Phonemic transcription & Word & Phonemic transcription \\
\hline hat & /'hæt/ & cult & /'kəlt/ & advantage & /əd 'væn təj/ \\
\hline book & /'buk/ & dare & /'der/ & behavior & /bi' he vyar/ \\
\hline pen & $/ \mathrm{p} \varepsilon \mathrm{n} /$ & fate & /'fet/ & circumstance & /'sər kəm stæns/ \\
\hline rat & /'ræt/ & guess & /'ges/ & defiance & /də'fai əns/ \\
\hline rug & /'r $\Lambda \mathrm{g} /$ & hint & /'hint/ & fantasy & /'fæn tə si// \\
\hline pig & /'pIg/ & $\operatorname{mood}$ & /'mud/ & interim & /'In tə rəm/ \\
\hline \multirow[t]{6}{*}{ hen } & /'hen/ & oath & /'o $\theta /$ & misery & /'mI zə ri/ \\
\hline & & plea & /'pli/ & narrowness & /'næ ro nes/ \\
\hline & & rush & /'r $\Lambda \int /$ & occasion & /ə 'ke 3ən/ \\
\hline & & truce & /'trus/ & protocol & /'pro tə kal/ \\
\hline & & verb & /'vərb/ & ridicule & /'ri də kyul/ \\
\hline & & zeal & /'zil/ & upheaval & /əp 'hi vəl/ \\
\hline \multicolumn{2}{|c|}{ Set 7 (simple short) } & \multicolumn{2}{|c|}{ Set 8 (simple long) } & \multicolumn{2}{|c|}{ Set 9 (complex long) } \\
\hline Word & Phonemic transcription & Word & Phonemic transcription & Word & Phonemic transcription \\
\hline basement & /'bes mənt/ & control & /kən 'trol/ & accident & /'æk sə dənt/ \\
\hline conscience & /'kan Jəns/ & dispute & /di 'spiut/ & clarinet & /kle rə' net/ \\
\hline discharge & /'dis čarg/ & himself & /him 'sclf/ & discipline & /'di sə plin/ \\
\hline entrance & /'en trəns/ & imprint & /'Im print/ & exception & /ək 'sep Jən/ \\
\hline falsehood & /'fals hud/ & junction & /'j $\Lambda \mathrm{nk} \int \partial \mathrm{n} /$ & hexagon & /'hek sə gan/ \\
\hline household & /'haus hold/ & mixture & /'mIks čər/ & industry & /'In də stri/ \\
\hline grievance & /'gri vans/ & prefix & /'pri fiks/ & protocol & /'pro tə kal/ \\
\hline mistress & /'mIs trəs/ & respect & /rə'spekt/ & ridicule & /'rI də kyul/ \\
\hline sheepskin & /'Jip skin/ & stipend & /'stai pend/ & specimen & /'spe sə mən/ \\
\hline traction & /'træk Jən/ & trumpet & /'trsm pət/ & tradition & 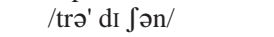 \\
\hline
\end{tabular}

Note. Sets 1 and 2 were also used in Experiment 2 of Caplan et al. (1992). Sets 3 and 4 were also used in Experiment 3 of Caplan et al.

Received April 20, 2001

Revision received December 11, 2002

Accepted January 27, 2003 